\title{
Designing blockchain systems to prevent counterfeiting in wine supply chains: a multiple-case study
}

\author{
Pamela Danese and Riccardo Mocellin \\ Department of Management and Engineering, University of Padova, \\ Padova, Italy, and \\ Pietro Romano \\ Polytechnic Department of Management and Architecture (DPIA), \\ University of Udine, Udine, Italy
}

\begin{abstract}
Purpose - The purpose of this paper is to contribute to the debate on blockchain $(\mathrm{BC})$ adoption for preventing counterfeiting by investigating $\mathrm{BC}$ systems where different options for $\mathrm{BC}$ feeding and reading complement the use of $\mathrm{BC}$ technology. By grounding on the situational crime prevention, this study analyses how $\mathrm{BC}$ systems can be designed to effectively prevent counterfeiting.

Design/methodology/approach - This is a multiple-case study of five Italian wine companies using $\mathrm{BC}$ to prevent counterfeiting.

Findings - This study finds that the desired level of upstream/downstream counterfeiting protection that a brand owner intends to guarantee to customers through $\mathrm{BC}$ is the key driver to consider in the design of $\mathrm{BC}$ systems. The study identifies which variables are relevant to the design of feeding and reading processes and explains how such variables can be modulated in accordance with the desired level of counterfeiting protection.

Research limitations/implications - The cases investigated are Italian companies within the wine sector, and the $\mathrm{BC}$ projects analysed are in the pilot phase.

Practical implications - The study provides practical suggestions to address the design of $\mathrm{BC}$ systems by identifying a set of key variables and explaining how to properly modulate them to face upstream/downstream counterfeiting.

Originality/value - This research applies a new perspective based on the situational crime prevention approach in studying how companies can design $\mathrm{BC}$ systems to effectively prevent counterfeiting. It explains how feeding and reading process options can be configured in $\mathrm{BC}$ systems to assure different degrees of counterfeiting protection.
\end{abstract}

Keywords Blockchain, Case study, Counterfeiting, Supply chain

Paper type Research paper

\section{Introduction}

Some characteristics of blockchain (BC) technology - namely, immutability, transparency, traceability, data security and disintermediation - make it particularly appropriate for addressing the counterfeiting of physical products (Alzahrani and Bulusu, 2018; Galvez et al., 2018). Policymakers and public agencies are seeking to assess and exploit these potential benefits. The EU Intellectual Property Office, as part

(C) Pamela Danese, Riccardo Mocellin and Pietro Romano. Published by Emerald Publishing Limited. This article is published under the Creative Commons Attribution (CC BY 4.0) licence. Anyone may reproduce, distribute, translate and create derivative works of this article (for both commercial and noncommercial purposes), subject to full attribution to the original publication and authors. The full terms of this licence may be seen at http://creativecommons.org/licences/by/4.0/legalcode

Funding: This research is funded by Fondazione Cassa di Risparmio di Padova e Rovigo.

Designing blockchain systems

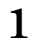

Received 7 December 2019

Revised 3 June 2020

14 October 2020

6 January 2021

Accepted 13 January 2021 
IJOPM

41,13

of a broad $\mathrm{EU}$ strategy to create a $\mathrm{BC}$ ecosystem, has recently launched a forum to encourage the development of $\mathrm{BC}$ implementations aimed at combatting counterfeiting (EUIPO, 2019). An additional aim is to determine whether BC could overcome the limits of existing anti-counterfeiting measures in ensuring compliance with food quality standards and EU directives, especially given that several recent food scandals (e.g. the UK's horsemeat incident and the USA's salmonella outbreak in peanut butter) indicate that mechanisms currently used are not sufficient to ensure food safety and integrity (Ali et al., 2017). These scandals have shaken consumers' confidence in the guarantees traditionally offered by the food sector, as demonstrated by a recent survey of Italian wine consumers, which showed that almost one-third of those surveyed had recently reduced their willingness to pay for wines with protected designation of origin (PDO) marks (Villano et al., 2017).

Several studies of BC pilot projects in different supply chain settings can be found in the literature (e.g. Martinez et al., 2019; van Hoek, 2019), but the investigation of the use of BC to prevent product counterfeiting is still limited, and most publications generally remain at the sense-making/exploratory stage or are mainly anecdotally descriptive, conceptual, purely technical or based on secondary data from single cases (Cole et al., 2019). Thus, as emerges from the systematic literature review by Queiroz et al. (2019), the assessment of the suitability of BC for addressing product counterfeiting represents an opportunity for SCM research. In particular, most existing research is based on the assumption that the technical characteristics of BC (such as data immutability, distributed consensus mechanisms and transparency) guarantee the accuracy and quality of the data saved and facilitate the ability to retrace the entire (true) transaction history for each product (e.g. Kumar et al., 2020; Martinez et al., 2019). This allows discovery of data manipulations and creates a transactional environment that is hostile to counterfeiting, where buyer-supplier information asymmetries that can favour counterfeiting are radically reduced (Schmidt and Wagner, 2019). Purchases are guaranteed by the BC itself (system trust), and trust in individuals and organisations is not required. Some authors warn about the validity of such assumption in real cases. Even though data are immutably saved on the BC, it is not designed to govern data acquisition, and $\mathrm{BC}$ feeding (the transferring of real-world data onto the $\mathrm{BC}$ ) is a critical security challenge for this type of application (Babich and Hilary, 2019; Creydt and Fischer, 2019). In particular, BC technology provides no protection against data that are intentionally manipulated prior to their validation on the network (Schmidt and Wagner, 2019). Moreover, reading information from the BC is an issue because the information accessed by consumers on a certain product may be stored offchain or may not actually refer to that specific product, given that commonly used smart labels can easily be cloned and then re-applied to counterfeited products (Lo et al., 2019; Kumar et al., 2020).

From the above, the core technical characteristics of $\mathrm{BC}$ technology do not necessarily assure effective counterfeiting protection if not complemented by a coherent set of measures for $\mathrm{BC}$ feeding and reading processes (i.e. a BC system). Although no study exists that offers an organic view of $\mathrm{BC}$ feeding/reading measures, scholars have described several options, ranging from artificial intelligence (AI) (Roeck et al., 2020) and using third-party certification bodies to verify input data (Creydt and Fischer, 2019), to the use of the IoT to ensure highquality and objective data collection (Kamble et al., 2019) in BC feeding and from the commonly used tagging technologies (e.g. barcodes and QR codes) to the highly secure smart labels, such as NFC tags, which use data encryption (Alzahrani and Bulusu, 2018) in BC reading. However, the literature still lacks a clear operationalization of the $\mathrm{BC}$ system construct, as well as a complete overview of all the relevant variables that can be used to design a BC system. Moreover, by mentioning the potential implementation issues of some choices (e.g. QR codes vs NFC tags or manual vs automatic data entry), previous studies (e.g. 
Galvez et al., 2018; Alzahrani and Bulusu, 2019; Azzi et al., 2019; Lo et al., 2019) implicitly suggest that the different feeding/reading options used to complement the core $\mathrm{BC}$ technological properties can assure protection against counterfeiting to different extents, but again the view remains fragmented.

Therefore, the present research intends to contribute to the debate on $\mathrm{BC}$ as a counterfeiting prevention measure by investigating the following research question (RQ): "How can BC systems be designed to effectively prevent counterfeiting?". In particular, this paper applies the situational crime perspective to study this phenomenon, as it can help in the exploration of counterfeiting mechanisms and the design of anti-counterfeiting measures by focusing on the reduction of physical opportunities for crime and the increase of opportunities for offender identification (Spink et al., 2013).

To answer the research question, we cross-analysed five case studies of $\mathrm{BC}$ adoption in the wine sector, which represents an ideal setting for various reasons. First, wine is one of the most counterfeited food products, as confirmed by several scandals, such as the Italian case of Brunello and Rosso di Montalcino (Villano et al., 2017) and the Côtes-du-Rhône fraud case in France (Vinex, 2020). Threats of lost revenue, image and customer trust have driven winemakers to adopt anti-counterfeiting measures and, in some pioneering cases, to launch exploratory pilot $\mathrm{BC}$ projects. Second, BC feeding and reading are crucially important in the wine industry, as counterfeiting can pose serious health risks for customers who depend on assurances of the origin and authenticity of products they buy. Third, these guarantees of product origin and compliance to process specifications are also essential for intermediaries - such as wine importers, distributors, resellers, specialized wine shops and hotel and catering service providers - since counterfeited products detected by customers are among the major causes of legal disputes or damage to companies' reputations.

This research contributes to the $\mathrm{BC}$ research field in several ways, providing interesting implications for academics and practitioners. First, by starting from the BC literature and comparing real $\mathrm{BC}$ implementations, it identifies the critical variables characterizing a $\mathrm{BC}$ system and assesses the ability of the different feeding and reading options in protecting against counterfeiting. This is novel in the $\mathrm{BC}$ field, as most previous studies, although acknowledging that there are different options for $\mathrm{BC}$ feeding and reading (e.g. Alzahrani and Bulusu, 2018; Kamble et al., 2019), focus on the features of $\mathrm{BC}$ technology (such as immutability, transparency and data security) and their value in guaranteeing system trust. This research instead helps to develop a clear operationalization of the $\mathrm{BC}$ system construct, which is lacking in the literature, and a more in-depth understanding of the level of counterfeiting protection provided by different BC system options. As recommended by several studies (Risius and Spohrer, 2017; Constantinides et al., 2018; Pereira et al., 2019), this research is not limited to the technical aspects of $\mathrm{BC}$ feeding/reading (e.g. smart labels or $\mathrm{AI}$ ) but considers both technology- and process-related decisions in designing a $\mathrm{BC}$ system and their power in countering counterfeiting. Finally, this research contributes to the wider debate on the efficacy of different anti-counterfeiting measures (see, for example, Apte and Petrovsky, 2016; Biswas et al., 2017; Alzahrani and Bulusu, 2018), laying the basis for a more rigorous and objective comparison between existing solutions and BC.

The remainder of this paper is organized as follows: Section 2 presents some theoretical underpinnings of the design of anti-counterfeiting measures, an overview of approaches to preventing product counterfeiting and a review of the relevant literature on $\mathrm{BC}$ systems. The research methodology is explained in Section 3; case analyses follow in Section 4. Section 5 discusses the results and provides theoretical and managerial implications. Finally, Section 6 summarizes the study findings and limitations and outlines further research opportunities.

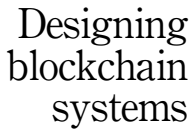


IJOPM

41,13

\section{Literature review}

\subsection{Designing anti-counterfeiting measures: theoretical underpinnings}

In the literature, various definitions and nuances of product counterfeiting can be found, such as adulteration, tampering and simulation (Spink et al., 2013). Counterfeiting also includes cases where fraudsters do not follow the regulatory definitions of good manufacturing practices (GMPs), good agricultural practices (GAPs) or good hygiene practices (GHPs). According to the World Trade Organization (WTO, 2020), counterfeiting is characterized as the misrepresentation of the identity or the source of products to deceive the purchaser into believing that he/she is buying the original goods. While the literature provides a wide variety of measures to mitigate counterfeiting (see Section 2.3), there is little theoretical development of the "chemistry" of the counterfeiting phenomenon, with the exception of the situational crime prevention approach (Spink et al., 2013). According to this theory, it is by restricting the offender's opportunities for infringement (i.e. addressing the "fraud opportunity structure") that an effective prevention of counterfeiting can be fulfilled. Thus, anti-counterfeiting measures should be designed by considering to what extent the decision-maker intends to suppress the impulse to offend. The effectiveness of measures to prevent counterfeiting can be evaluated by considering that crime is the result of a choice: more effective measures are those that deter or detect intentional infringements because offending appears less feasible or attractive (for instance, because it is easier to intercept a counterfeited product or to identify the counterfeiter). Basically, the situational crime prevention approach suggests two partially overlapping ways of restricting the offender's opportunities: (1) physically reducing opportunities (e.g. steering wheel locks are a good visual deterrent for car thieves and home security systems discourage burglaries) and (2) increasing the chances of being identified (e.g. the obligation to wear crash helmets significantly reduced thefts of motor-cycles, as thieves could be easily spotted by the police).

Situational crime prevention also establishes that the understanding of the fraud opportunity structure requires a wide-ranging awareness of the vulnerabilities of the various supply network processes, ideally including sourcing, production, distribution, retail and, in extreme situations, final disposal or reuse of packaging material (Speier et al., 2011; Spink et al.,2013). Since the fraud opportunity structure is significantly different in the steps before vs those after the producer sells the product, in this paper, we distinguish between "upstream counterfeiting", which concerns sourcing and production activities, and "downstream counterfeiting", which concerns distribution and retail activities. Noncompliance with compulsory international or national production specifications and with product and production process characteristics declared by the company (e.g. use of vulnerable workers, failure to employ of bio, vegan, eco-friendly agricultural or production practices) are forms of upstream counterfeiting. Some practical cases are the production of drugs with no or few active ingredients or the use of substandard aftermarket parts for automobiles or aircrafts (Hopkins et al., 2003). In downstream counterfeiting, the fraud opportunity structure is different. For instance, in the wine industry, counterfeiting can emerge after the wine company sells a bottle and takes the form of fake labels, bottle relabelling or refilling with cheaper wines. The recent large-scale wine scam uncovered by French anti-fraud bodies, in which a merchant tried to sell more than 48 million litres of wine falsely labelled as Côtes-du-Rhône, is an example of downstream counterfeiting (Vinex, 2020).

The situational crime prevention perspective was applied to our research setting where the decision-makers are brand owners who use $\mathrm{BC}$ as a measure that deters or detects intentional infringements and also as a traceability and communication tool that allows customers to autonomously verify that the products they buy are not affected by upstream and/or downstream counterfeiting. In this setting, the prevention of counterfeiting is pursued by deterring upstream supply network members from non-adherence to origin, production or 
quality control specifications (both compulsory and declared) and deterring downstream members from manipulating original goods during distribution and retail. Thus, in this paper, we assume that the desired level of upstream or downstream counterfeiting protection the brand owner intends to guarantee to customers through $\mathrm{BC}$, by setting precise requirements in terms of restriction of the offender's fraud opportunity structure, is the key driver in the design of their $\mathrm{BC}$ systems.

\subsection{Measures to prevent product counterfeiting}

We identify three broad categories of approaches that companies use to mitigate product counterfeiting: product/packaging-related, customer information/education-related and process-related measures. Table 1 offers a short description of each measure together with its weaknesses and classifies each of these on the basis of its suitability to address upstream and/or downstream counterfeiting.

Like track-and-trace systems, $\mathrm{BC}$ can be considered a measure to prevent both upstream and downstream counterfeiting (Schmidt and Wagner, 2019). Unlike the other measures and in common with traditional track-and-trace systems, it allows customers to autonomously verify the authenticity of each product without the need to involve specific equipment/ competencies or perform destructive chemical/physical/organoleptic analyses. The literature emphasizes BC's capability to overcome the limits of traditional track-and-trace systems (e.g. risk of data manipulation or the poor quality of input data) thanks to its inherent features, such as transparency and decentralized and immutable data storage (Alzahrani and Bulusu, 2019; Saberi et al., 2019). However, since companies adopt different BC feeding and reading options (Section 2.3), a precise and objective comparison between $\mathrm{BC}$ and existing counterfeiting approaches, in particular track-and-trace systems, would require a clearer understanding of the different $\mathrm{BC}$ system configurations and their advantages compared to traditional track-and-trace systems.

\subsection{Blockchain systems}

Although most studies recognise that BC's potential for preventing counterfeiting depends on its technical characteristics (immutability, transparency, etc.) (Wang et al., 2019), a growing number of authors are discussing different $\mathrm{BC}$ feeding and reading options to complement $\mathrm{BC}$ technology and their implications for counterfeiting. A major issue concern is ensuring the quality of the input data. According to Creydt and Fischer (2019) and Bumblauskas et al. (2020), there is currently no universal solution for BC feeding. The potential of $\mathrm{BC}$ technology for preventing product counterfeiting can be improved by additional security measures and associated technologies that protect against the risk of human error and potentially dishonest behaviours, thus ensuring that the data that are entered into the BC are of a higher quality (van Hoek, 2019). Drawing on the assumption that information captured by humans can be subjective and unreliable, Roeck et al. (2020) suggest pairing $\mathrm{BC}$ with $\mathrm{AI}$ to identify patterns across manually entered data that may indicate anomalies or inconsistencies. The integration of $\mathrm{BC}$ and $\mathrm{AI}$ is recommended by several SCM scholars, although the empirical evidence for a combination of these technologies is still limited to a few early use cases (Kshetri, 2018; van Hoek, 2019; Wamba and Queiroz, 2020). Other studies consider the involvement of neutral third-party certification bodies to carry out field audits and verify data quality before they are manually saved (Creydt and Fischer, 2019). Commenting on this feeding measure, Kamilaris et al. (2019) argue that the need for such intermediaries may compromise the building of decentralized trust for BC technology. An increasing number of studies suggest the use of IoT sensors to automatically enter data to ensure that the feeding process remains objective by eliminating human interaction and the risk of input errors or fraudulent behaviours (Creydt and Fischer, 2019; Kamble et al., 2019;

\section{Designing blockchain systems}




\begin{tabular}{l} 
IJOPM \\
41,13 \\
$\mathbf{6}$ \\
\hline
\end{tabular}

Table 1.

Traditional anticounterfeiting approaches

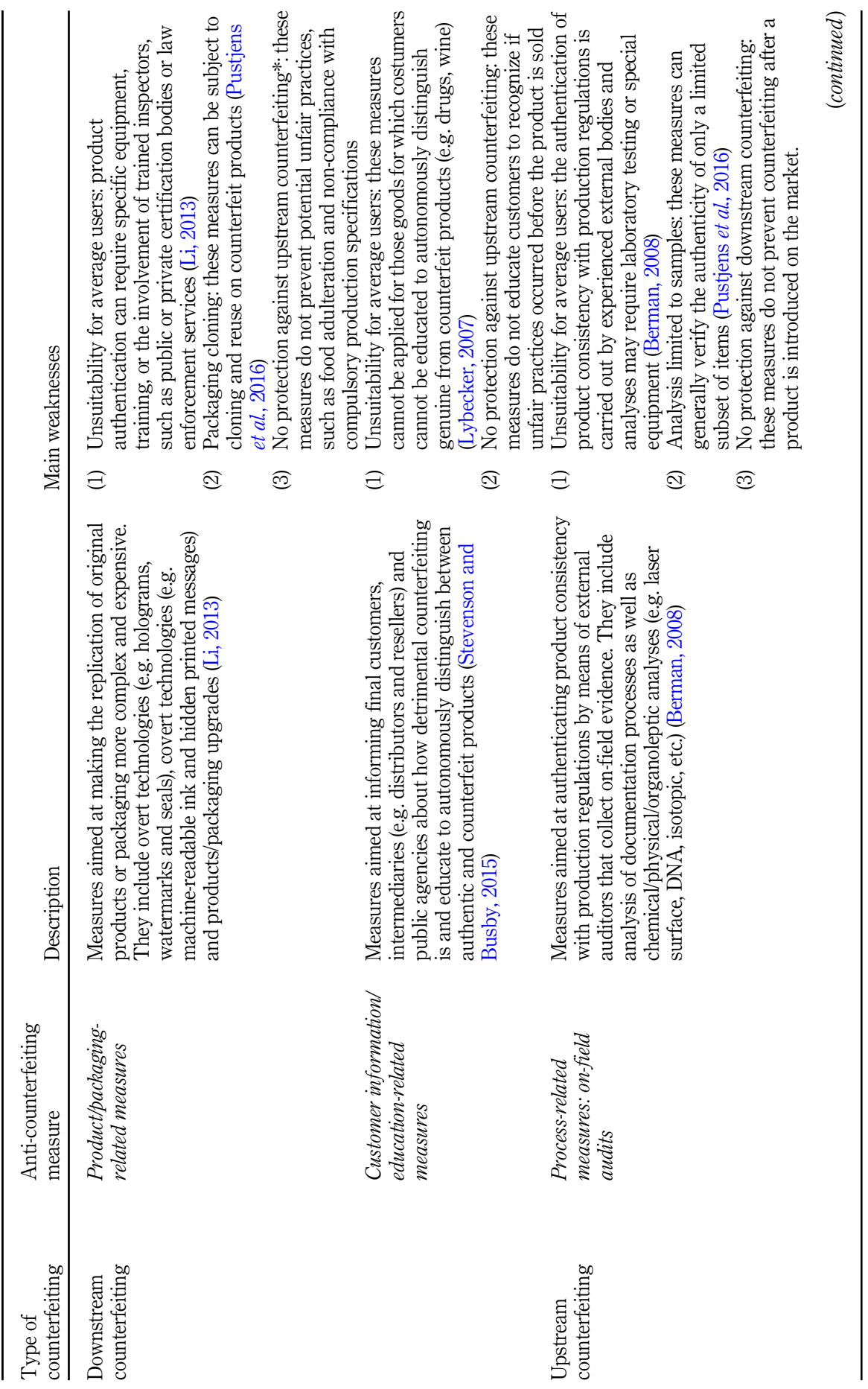




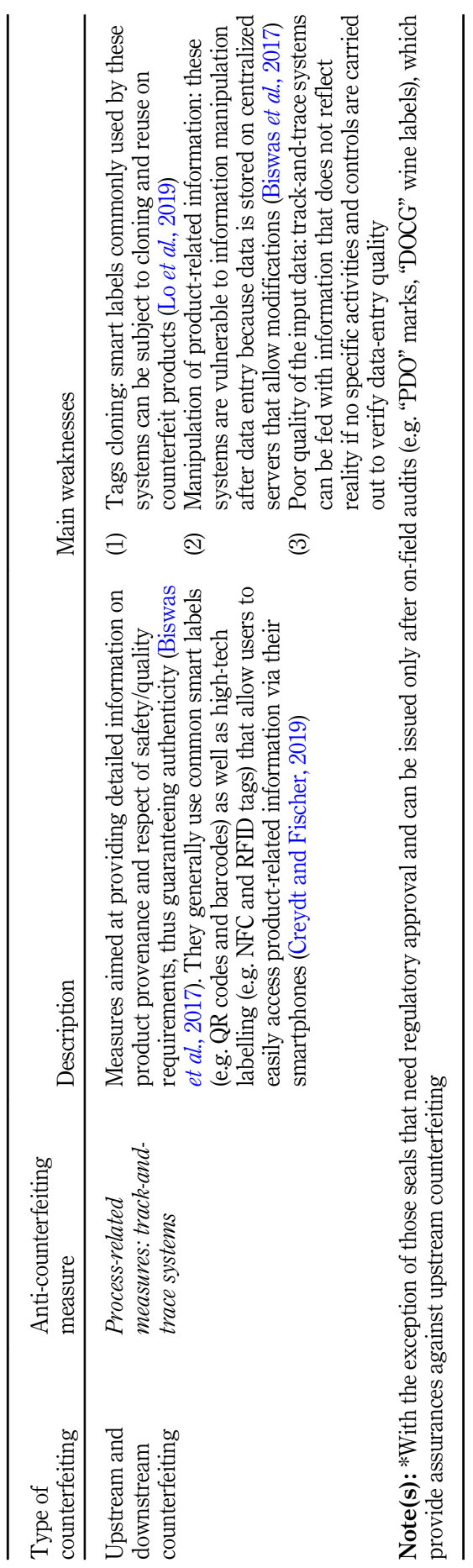

Designing

blockchain

systems

7

Table 1. 
IJOPM

41,13

8

Bumblauskas, 2020). Moreover, the IoT would eliminate the delay between data collection and data recording, which is an issue for manual entry (Zelbst et al., 2019). In this last case, periodical data transmission after data collection is a common practice to avoid network congestion and higher transaction costs (Zhang et al., 2020).

A further set of options and decisions is related to the security of smart labels that connect physical products to the related information saved on the BC (i.e. BC reading). Lo et al. (2019) point out that commonly used smart labels - such as barcodes, QR codes and serial numbers - are vulnerable to attacks, e.g. cloning. In their study, Azzi et al. (2019) argue that RFID tags can also be easily cloned, despite their more technologically advanced nature. This view is supported by Lo et al. (2019), who maintain that cloned tags contribute to the circulation of counterfeited products in supply chains. Conversely, as outlined by Alzahrani and Bulusu (2018), NFC labels provide greater guarantees of tag cloning and tampering. The security of NFC tags against tampering and replication can be improved using data encryption, although it is an expensive measure (Alzahrani and Bulusu, 2019).

A final critical point related to $\mathrm{BC}$ reading, although under-investigated, is that in real cases $\mathrm{BC}$ systems usually treat the $\mathrm{BC}$ as a backend database behind a centralized web server (Singhal et al., 2018). Although an ideal application that prevents potential manipulations should make information visible to final customers directly from the $\mathrm{BC}$ hence ensuring complete decentralization, information displayed through user-friendly web pages or mobile applications is usually stored on external servers that should ideally reflect what is written on the $\mathrm{BC}$. To let customers verify the alignment between the communicated information and that saved on the $\mathrm{BC}$, companies associate this information with the related $\mathrm{BC}$ transaction link where the information was originally saved (Montecchi et al., 2019). As clarified by $\mathrm{Xu}$ et al. (2019), large files (e.g. photos, videos or pdfs) are not stored on the BC due to the technology's limited capacity for handling a large quantity of data; therefore, it is usually preferred to store hash values, namely fixed-length alphanumerical strings unequivocally associated with each document by a secure encryption algorithm. The hash value allows verifying whether the information displayed on the web pages or mobile applications has been manipulated because any change in off-chain data would result in a different hash value from that saved on the BC.

Table 2 summarizes the different options that, according to the literature, could be chosen for $\mathrm{BC}$ feeding/reading and some open issues. Although use cases and $\mathrm{BC}$ studies contemplate the existence of different configuration choices for $\mathrm{BC}$ feeding/reading, they all lack a holistic and in-depth understanding of how a brand owner can design a $\mathrm{BC}$ system to assure a certain degree of security in preventing counterfeiting. In particular, there is uncertainty about which variables are relevant to the design of feeding and reading processes and how such variables can be modulated in accordance with the desired counterfeiting protection level. To address these gaps, the present research applies the concept of restriction of the fraud opportunity structure to the field of $\mathrm{BC}$ design to unveil the relationships between the degree of protection against counterfeiting a brand owner intends to guarantee to customers and the feeding/reading choices in the design of $\mathrm{BC}$ systems.

\section{Research methodology}

Considering the lack of empirical research on the use of $\mathrm{BC}$ to prevent counterfeiting, a qualitative and exploratory approach was chosen for the study. According to Yin (2017), when addressing "how" questions and examining recent or contemporary events, a multiplecase study is a particularly good methodology. Moreover, the purpose of this research is theory building, and research based on case studies facilitates the full understanding of a reallife complex phenomenon in its natural setting as well as the identification of its critical variables and the linkages between them (Yin, 2017). 
Feeding/

Reading

Feeding
Options and issues

Data collected and uploaded manually, prone to unintentional human errors, intentional dishonest behaviours and delays (Galvez et al., 2018; Azzi et al., 2019; Creydt and Fischer, 2019; Kamble et al., 2019; Kamilaris et al., 2019; Montecchi et al., 2019; Schmidt and Wagner, 2019; van Hoek, 2019; Zelbst et al., 2019; Bumblauskas et al., 2020). Complementary measures are

(1) AI to cross-check data (Kshetri, 2018; Montecchi et al., 2019; van Hoek, 2019; Bumblauskas et al., 2020; Roeck et al., 2020)

(2) Neutral third-party bodies to verify data quality (Creydt and Fischer, 2019) Data automatically captured and uploaded on BC by IoT sensors, lowering the risk of input error, unethical behaviours and delays (Azzi et al., 2019; Creydt and Fischer, 2019; Kamble et al., 2019; Lo et al., 2019; Zelbst et al., 2019; Bumblauskas et al., 2020)

Connection between physical products and related information on $\mathrm{BC}$ through: common tagging techniques (barcodes, serial numbers, QR codes, RFID) vulnerable to cloning (Azzi et al., 2019; Lo et al., 2019), or more sophisticated tagging techniques - such as NFC with data encryption - that prevent cloning (Alzahrani and Bulusu, 2018, 2019)

Communication to customers: information can be read through websites or mobile applications (Creydt and Fischer, 2019; Montecchi et al., 2019)

Information presented to customers can be stored off-chain on a centralized server (Singhal et al., 2018). Customers can access the associated BC transaction (Montecchi et al., 2019)
Designing blockchain systems

Table 2.

$\mathrm{BC}$ system configuration

\subsection{Case selection}

We selected cases based on literal and theoretical replication (Yin, 2017). We deliberately searched for companies that showed differences and similarities in terms of the adopted BC systems (e.g. type of smart label, control measures over data, etc.). Furthermore, we attempted to include companies in our sample that adopted $\mathrm{BC}$ systems developed by different technological partners currently offering $\mathrm{BC}$ systems for the wine sector to present a broader picture of the phenomenon and facilitate the generalization of the results. To identify the cases, we first compiled a list of all the $\mathrm{BC}$ systems offered by the technology providers in the wine sector in Italy and of the winemakers involved in the implementation of these solutions. This was made possible by Internet searches and our attendance at several conferences and workshops related to $\mathrm{BC}$ technology. Second, we collected publicly available data and information on each $\mathrm{BC}$ system to decide whether it might represent an interesting case for the study.

We contacted all technology providers to present the area and purpose of the study, the research team and a brief outline of the interview protocol, and then ascertained their willingness to participate in the study. Subsequently, we identified some winemakers that were exemplar cases in the adoption of $\mathrm{BC}$ systems and contacted the CEOs of these winemakers via email, again presenting the team and research and ascertaining their willingness to collaborate. We also promised to give both the technology providers and winemakers the final study report and guaranteed data confidentiality. In the end, all technology providers and five Italian winemakers - pioneers in the adoption of $\mathrm{BC}$ technology in the wine sector - agreed to participate in our study. We will refer to the cases and related winemakers with labels from $\mathrm{A}$ to $\mathrm{E}$.

Ideally, the unit of analysis for the study would be the supply network involved in the BC system. However, like the majority of $\mathrm{BC}$-based projects at the time of this writing, those selected are in a pilot phase and therefore involve a limited number of actors, as shown in Table 3. In particular, companies $\mathrm{C}, \mathrm{D}$ and $\mathrm{E}$ are fully integrated winemakers, as they are responsible for all production activities from grape harvesting to bottling. Companies A and $B$ rely on external grape suppliers. 


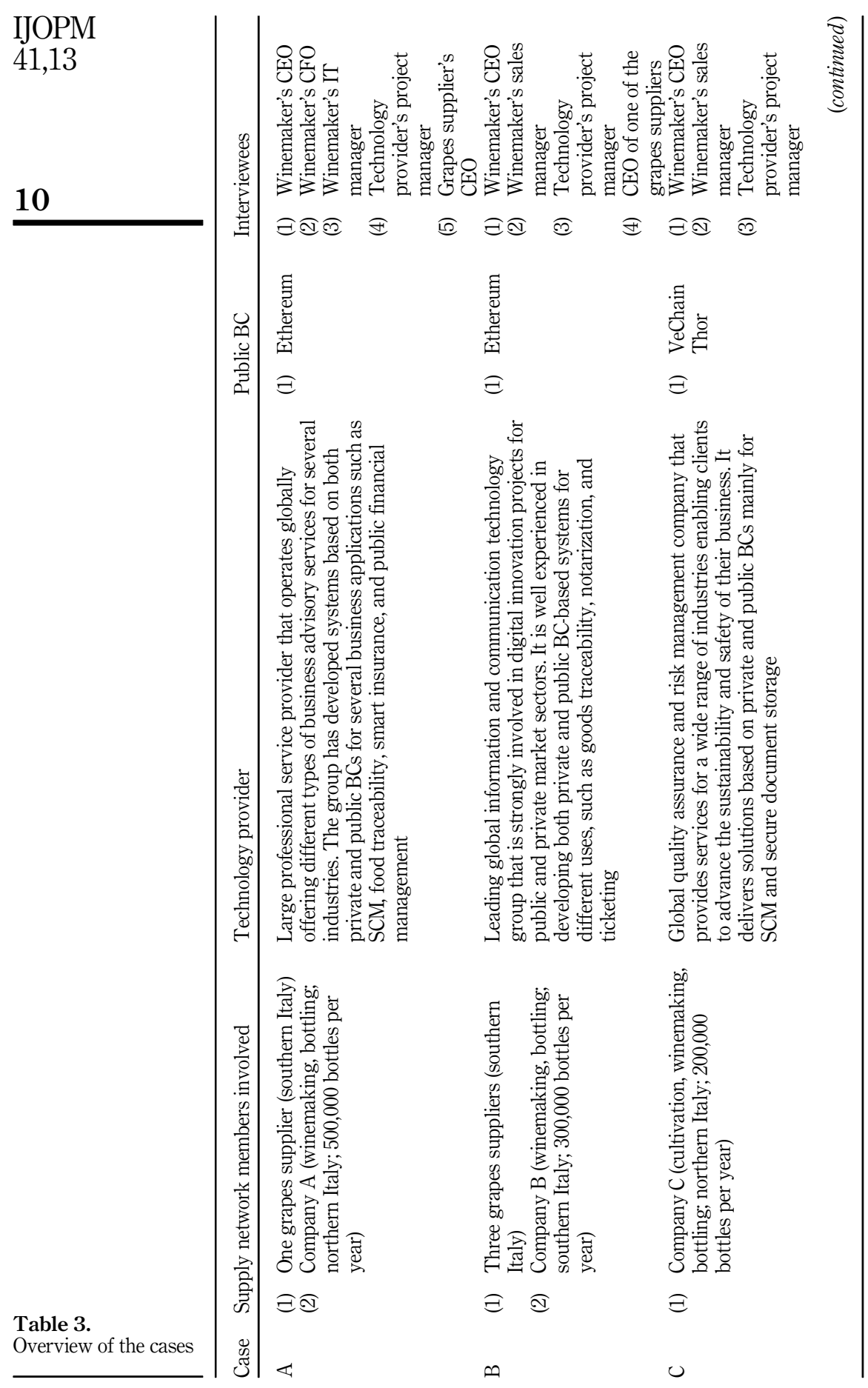




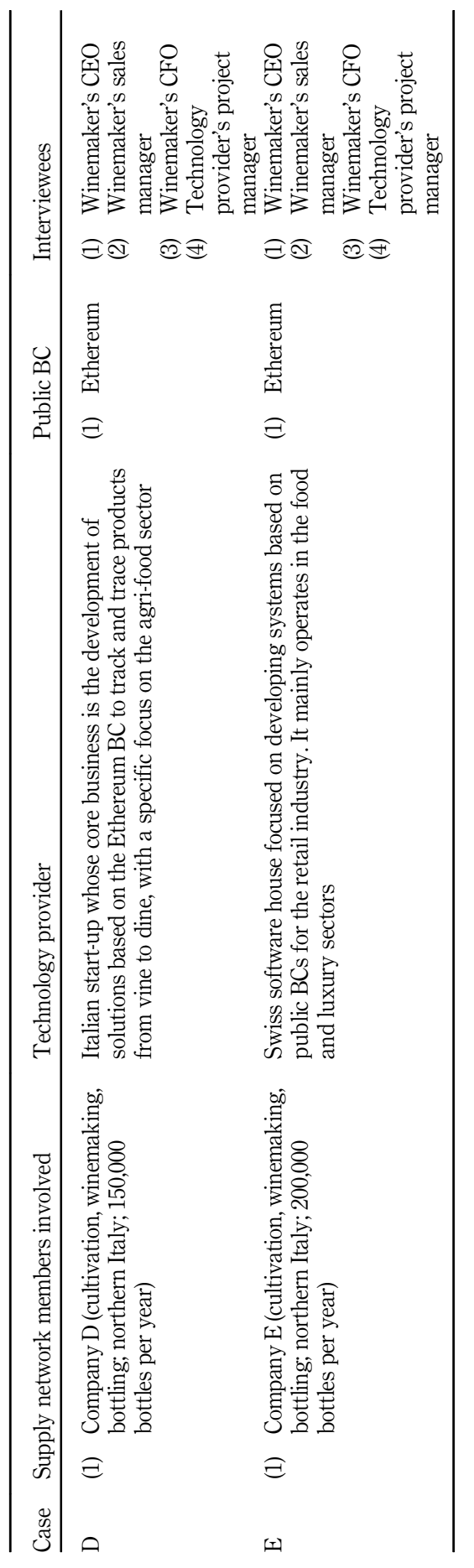

Designing

blockchain

systems

11

Table 3. 
IJOPM

41,13

3.1.1 Sample controls and research boundaries. The cases investigated belong to the wine sector and are Italian companies. Controlling for industry and country effects is important in this research, as they can limit choices in designing a secure $\mathrm{BC}$ system. In particular, the wine sector exhibits some peculiarities that are worth considering. Many processes are handcrafted, and the IoT, which in general helps to solve critical issues related to $\mathrm{BC}$ feeding (Table 2), is difficult to apply. More precisely, it is not suitable for determining whether viticulture and vinification processes are carried out under the requirements of production regulations as many parameters are hard to measure using sensors (e.g. the types of grapes used for vinification and their percentage as well as the type of oak used for ageing). Wine cellars are commonly underground where connectivity is poor and the environment not ideal for the functioning of electronic devices. For these reasons, the issue of $\mathrm{BC}$ feeding is particularly challenging in this sector, and finding possible solutions and alternatives is of paramount importance. Similar considerations apply in other industries, generally associated with agriculture, although some environmental parameters can be measured through sensors (Galvez et al., 2018), or in the luxury clothing industry where products are handmade, which makes the investigation and results elaborated in this research of interest in different contexts from the wine sector. In more capital-intensive industries that use automated production lines, such as the pharmaceutical industry, where the IoT can be extensively and easily used, the investigation and results elaborated in the present study are less applicable.

A further research boundary is that the cases investigated are pilot projects that involve few actors. This limits the opportunity to test which BC system choices, among those identified here for designing a secure $\mathrm{BC}$ system, are more appropriate in complex supply networks (see Section 6.1).

Finally, as pointed out in Table 3, all cases adopt well-known public BCs, namely Ethereum (4 cases) and VeChain Thor (1 case). Public BCs, compared to private BCs, maintain some core advantages, such as data immutability, decentralized governance, open network access and transaction visibility. Private BCs generally offer enhanced data privacy and transaction writing speed compared to public BCs (Viriyasitavat and Hoonsopon, 2019). Given these differences, we argue that this research is only generalizable to networks using public BCs because the use of private BCs for anti-counterfeiting and how they can be complemented with $\mathrm{BC}$ feeding and reading could significantly change.

\subsection{Data collection}

Data collection took place between October 2018 and May 2019. Semi-structured interviews were chosen as the primary data source because they represent a highly efficient way to gather rich empirical data (Eisenhardt and Graebner, 2007).

We collected information from multiple respondents (Table 3) to capture data from different perspectives, reduce respondents' biases and reach data and theoretical saturation (Bowen, 2008). We decided to interview the winemakers' CEOs. Even if they were not BC experts, they were all directly involved in the pilot projects and were best informed about issues, such as the context, the reasons leading to $\mathrm{BC}$ adoption and the expected results. As often happens (even in the more structured wine companies), the winemakers' CEOs also played operational roles in the viticulture and vinification processes as agronomists or oenologists and were also involved in promotion and sales. Then, for each case, we interviewed the project manager of the technological partner to ask for more specific details and empirical evidence to clarify the technical aspects of the $\mathrm{BC}$ systems. With the same aim, we also interviewed the IT manager of company A; this role was not present in the other companies. It is worth noting that, in the cases investigated, the decision to adopt a $\mathrm{BC}$ system came from the winemaker who, with the support of the technology provider, promoted and guided the $\mathrm{BC}$ project. Although they were not directly involved in the decisions about the 
design of the $\mathrm{BC}$ system, in cases $\mathrm{A}$ and $\mathrm{B}$, where the winemakers were not vertically integrated upstream, we also interviewed the CEOs of two grapes suppliers involved in the $\mathrm{BC}$ project. As is typical in the wine sector, they were vignerons, owners of the vineyards and agronomists expert in grape cultivation. They, therefore, represent key figures in the wine production processes, working closely with winemakers and holding crucial information on cultivation that might be of interest to customers. In case B, given the uniformity in the production activities and in the $\mathrm{BC}$ use, we had sufficient information to limit our interviews to one supplier.

We mainly collected data through face-to-face, on-site interviews. For logistical reasons, as suggested by Creswell (2013), we used video conferences with actors located in southern Italy (four interviewees). In total, considering all the respondents of firms and technology providers, 20 interviews were conducted for all cases.

To improve accuracy, each interview was first recorded and then transcribed. Field notes were taken by two researchers to reduce observer's bias. To enhance reliability and validity, an interview protocol was developed based on the existing literature and our research question (Yin, 2017) (see Supplementary File 1). Semi-structured interviews allowed us to create a balance between an open discussion characterized by mutual reflection and knowledge sharing and a more specific questionnaire-based conversation with the aim of finding evidence for research purposes. While all respondents were asked all the questions in the protocol, the level of detail of the discussion on the different points and the focus varied depending on the respondent (Pandey and Patnaik, 2014). For example, when interviewing representatives of the technological providers, we focused on the characteristics of the $\mathrm{BC}$ systems, whereas with the winemakers' CEOs, we focused on the firms' internal processes, competitive strategy, etc.

Each interview lasted on average from 90 to $120 \mathrm{~min}$. To validate the information or correct any misunderstandings, we decided to provide interviewees with a structured summary of the interviews' verbatim transcripts, including all relevant information for which feedback was necessary. Providing the respondent with this structured summary, instead of the entire transcript, maximized the probability of getting feedback from him/her, and thus minimized the risk of bias.

Whenever possible, triangulation of information from different sources was used to increase the reliability of the research and the validity of the analysis (Yin, 2017). In addition to interviews, the following secondary data sources were used: (1) official documents provided by the companies and technological partners (e.g. slides of internal presentations), (2) web resources (websites, public interviews) and (3) data gathered from the implemented $\mathrm{BC}$ systems. Follow-up phone calls were made to clarify any doubts about the data collected.

\subsection{Data analysis}

The analysis was carried out in two steps: analysis of within-case data and searching for cross-case patterns (Yin, 2017).

We started the within-case analysis by creating a detailed write-up of each case. Data reduction was then used to summarize the large amount of collected information: we broke down data and characterized each case based on a series of variables that described the different $\mathrm{BC}$ systems and the upstream and downstream counterfeiting protections (Tables 4 and 5). We applied the data coding procedure recommended by Yin (2017) to identify variables. As is typical in theory-building case-based research, this process was cyclic and iterative (Voss et al., 2016). Starting from existing research on counterfeiting and BC (Section 2), we identified the relevant concepts in this study and, in particular, based on BC literature, we identified a number of variables potentially useful to distinguish $\mathrm{BC}$ systems. While some differences between $\mathrm{BC}$ systems clearly emerged from the use cases and $\mathrm{BC}$

\section{Designing blockchain systems}


IJOPM
41,13

14

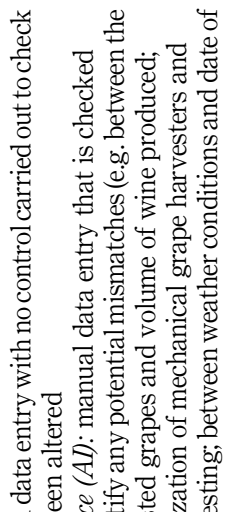

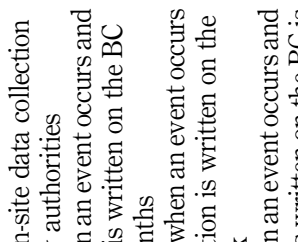

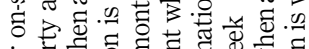

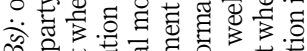

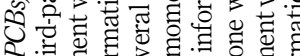

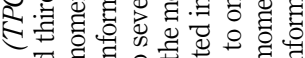

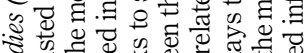
¿

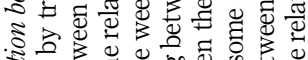

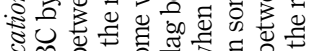

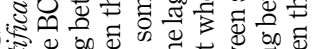

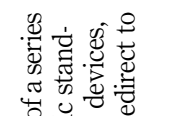

它氖

跑苋畐

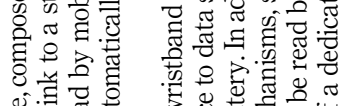

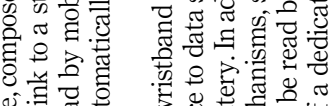

రृ:

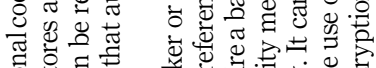

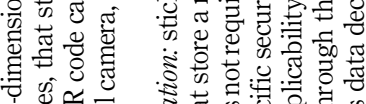

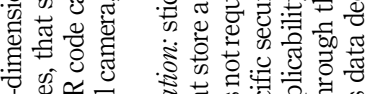

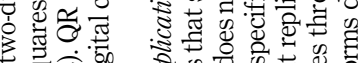

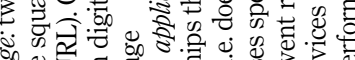

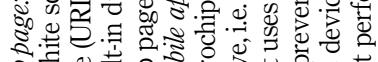

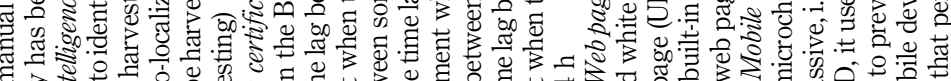

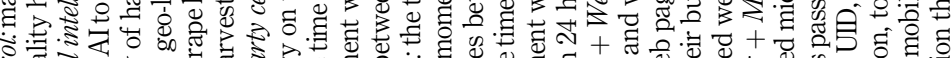

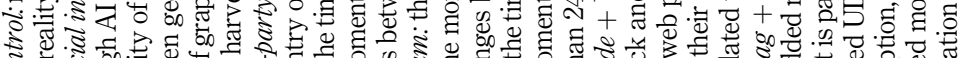

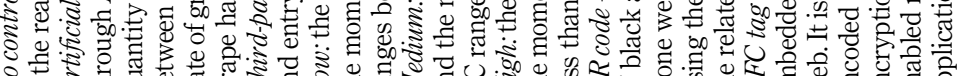

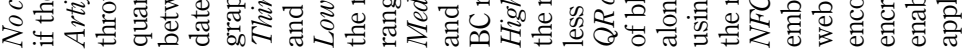

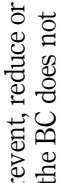

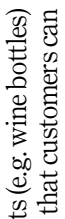

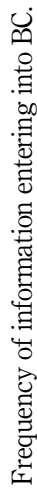

造包

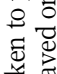

害芯

豞

壱茌

:总: 总

总:

ठै

흘

:

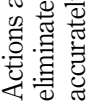

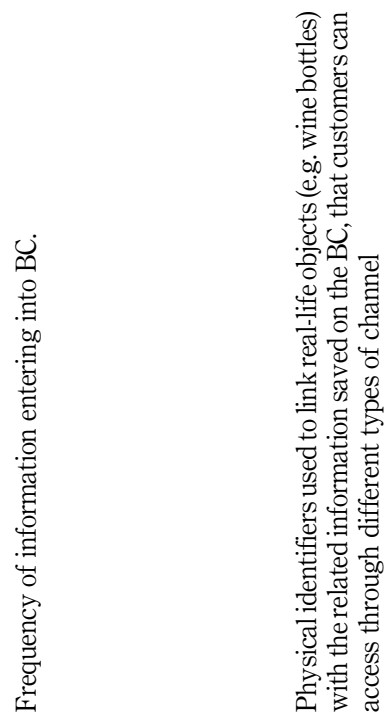

Table 4.

Data reduction and operationalization

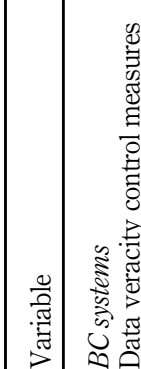

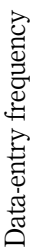

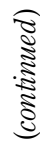




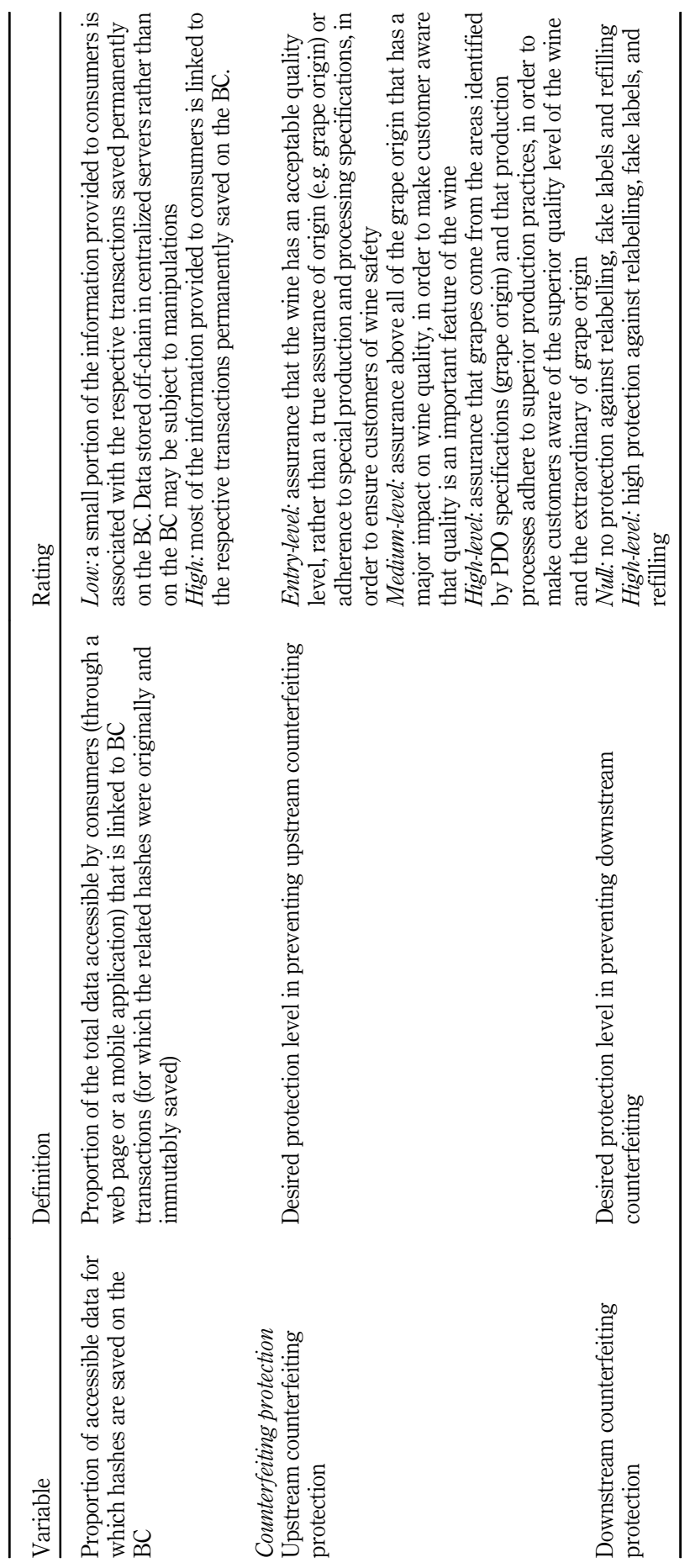

Designing

blockchain

systems

15

Table 4. 


\section{IJOPM \\ 41,13}

16

Table 5.

Within-case analysis $\mid$

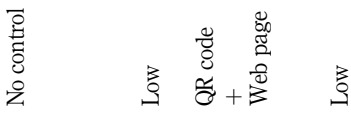

要

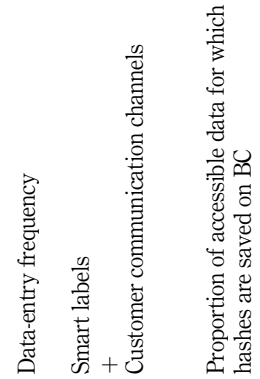

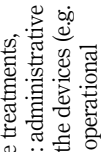

荧

क

항

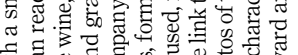

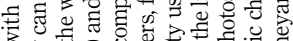

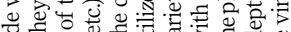
8 F

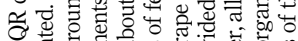

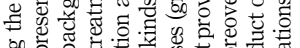
on 1. 赵.

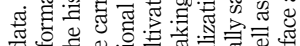

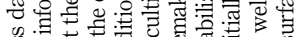

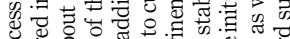

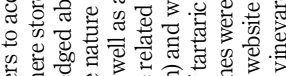

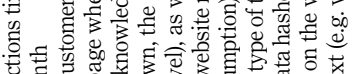

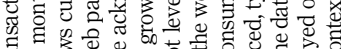
年

늘

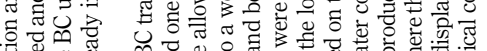

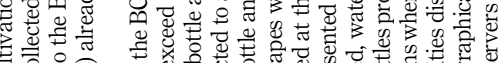

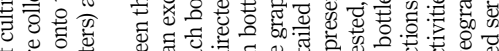

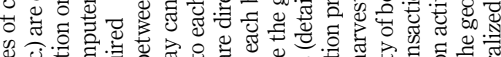
y

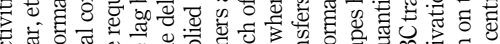

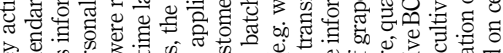

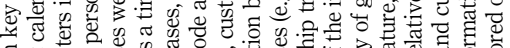
₹ 4 \%

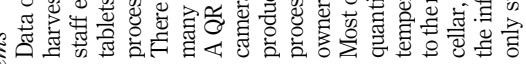

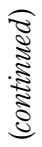
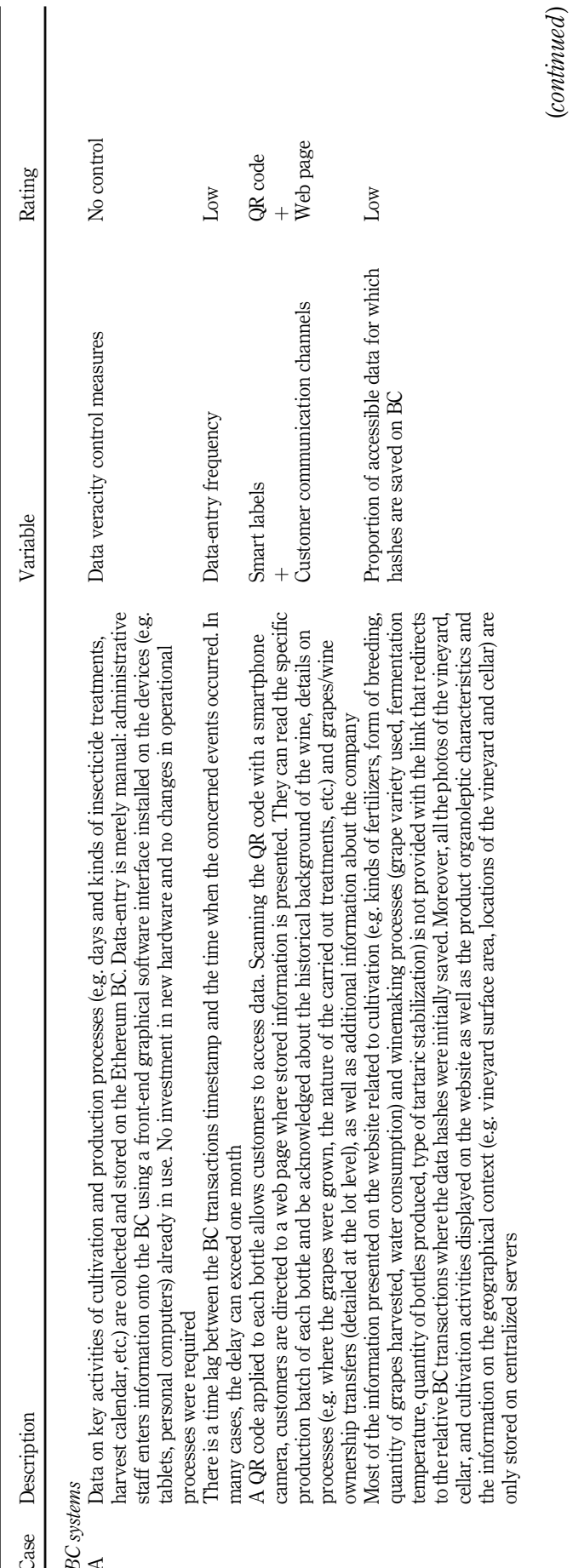


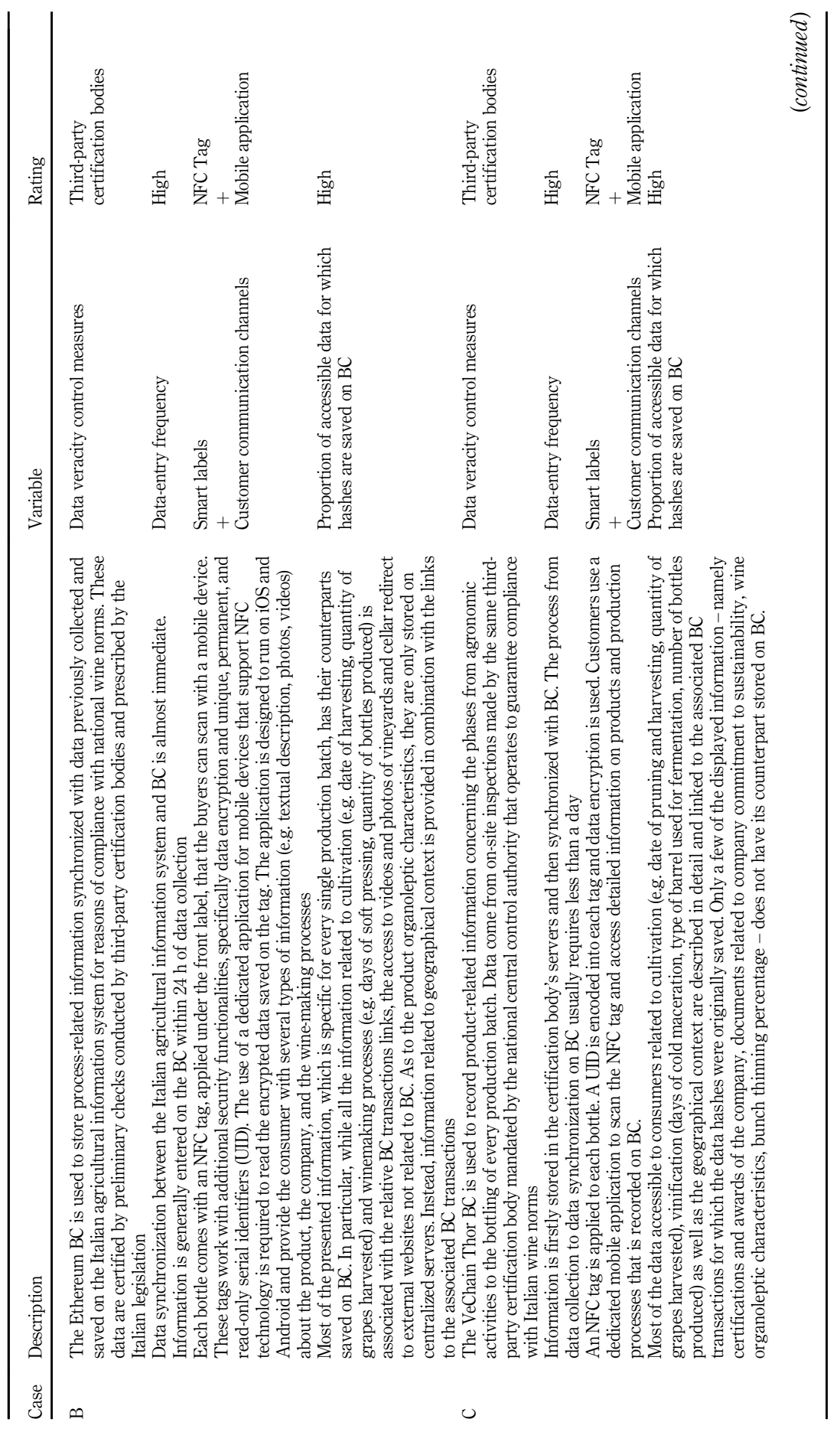

$\begin{array}{r}\text { Designing } \\ \text { blockchain } \\ \text { systems } \\ \mathbf{1 7} \\ \hline\end{array}$

Table 5. 


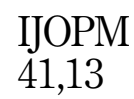

|

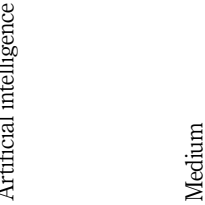

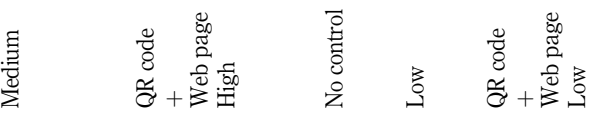

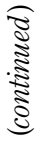

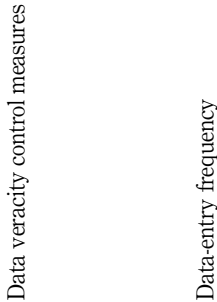

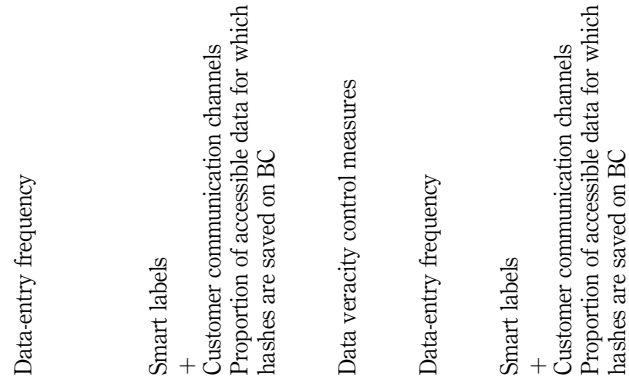

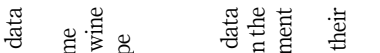

要

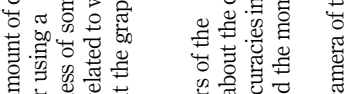

品芯芯

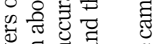

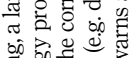

ô 0 过

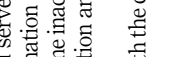

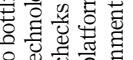

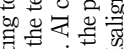

चु

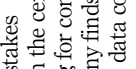

范

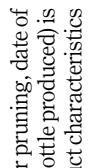

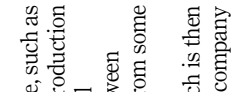

券密

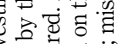

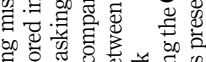

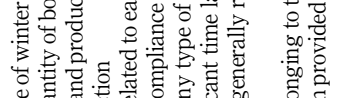

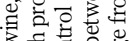

零

苞焉

害

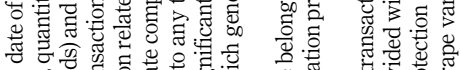

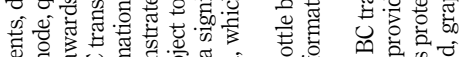

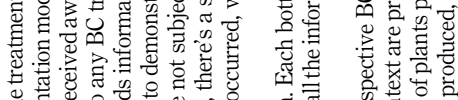

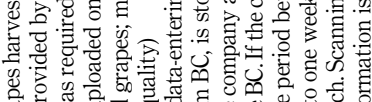

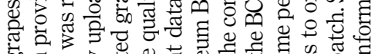

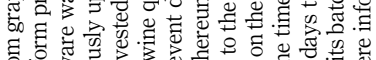

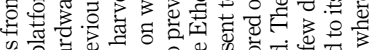

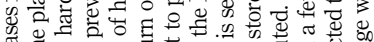

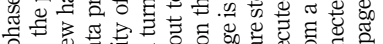

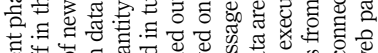

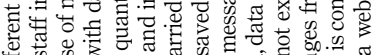

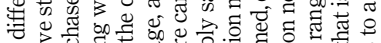

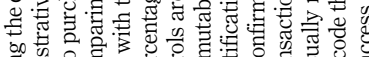

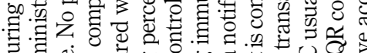

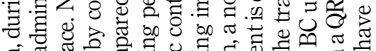

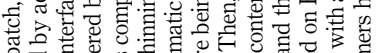

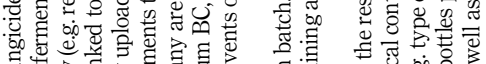

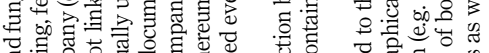

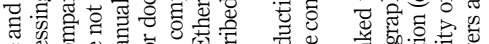

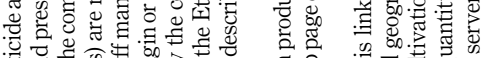

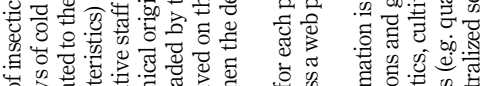

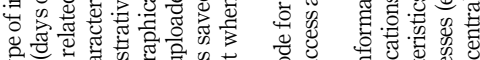

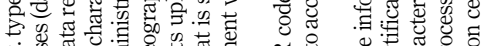
of ơ

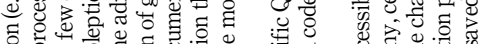

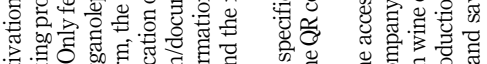
. : है

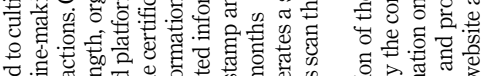

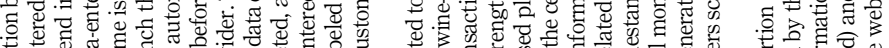

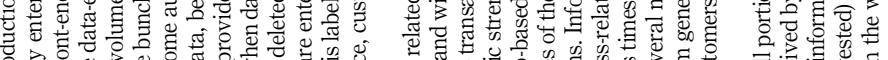

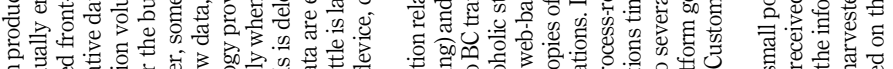

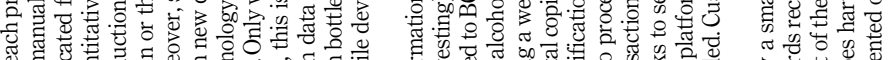
匹

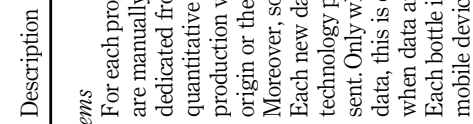

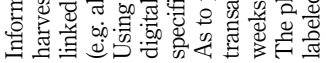

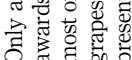

Table 5. 仓ो 


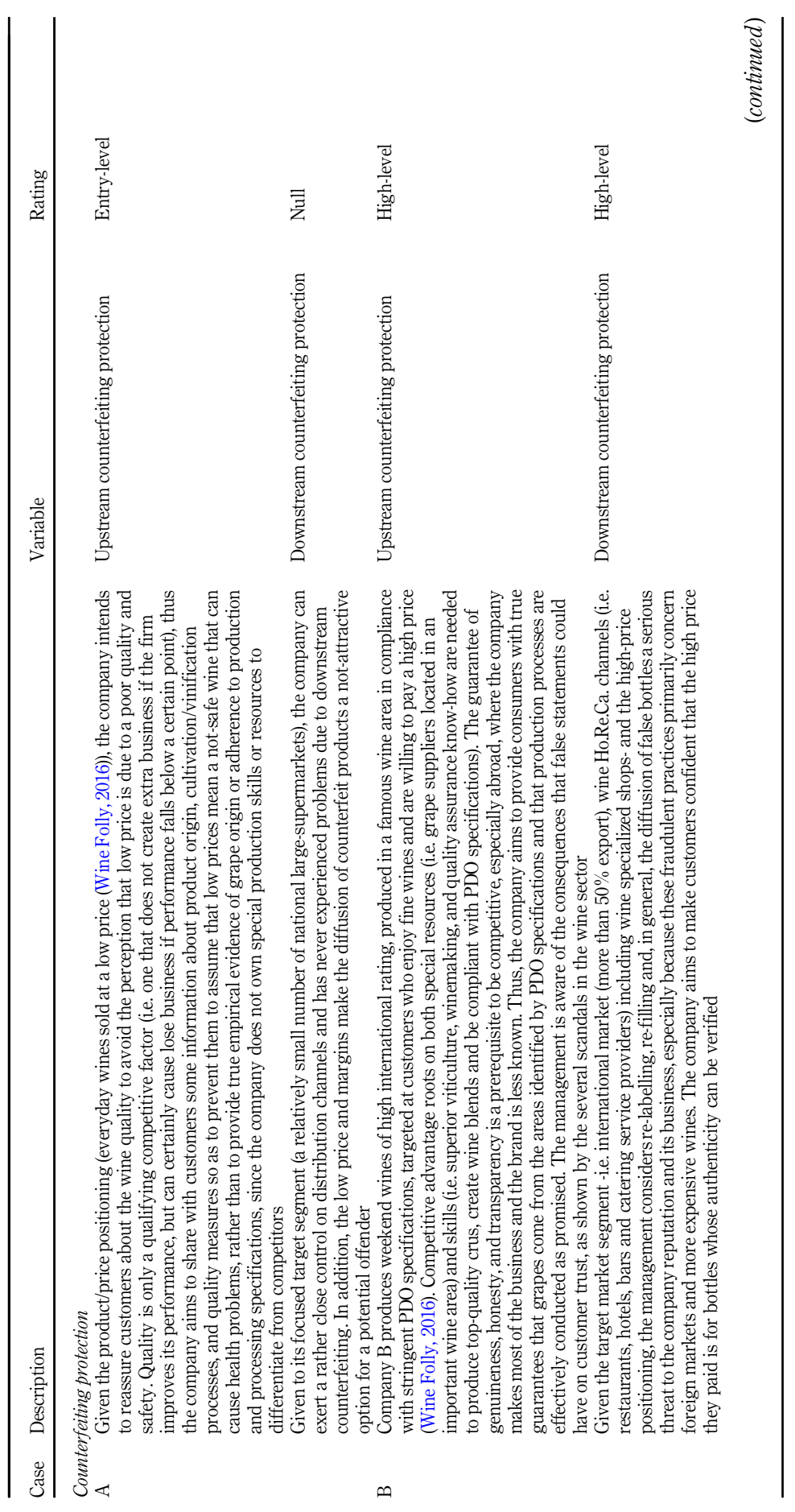

Designing
blockchain
systems

19

Table 5. 


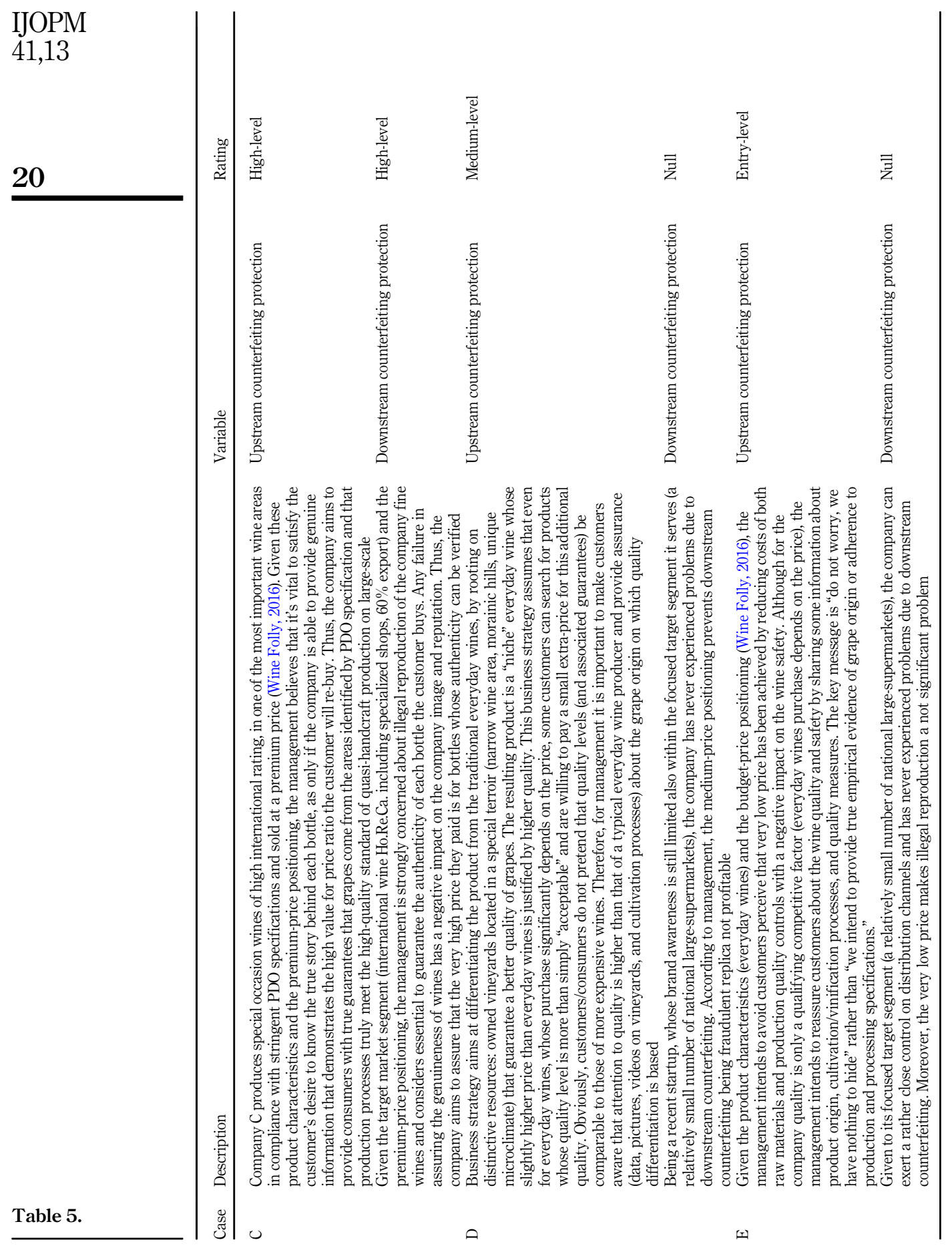


studies (Table 2), a clear operationalization of the $\mathrm{BC}$ system construct was lacking in the literature. Thus, looking at evidence emerging from the data was fundamental to abductively identifying relevant variables. Identified variables were rated according to precise rules, and again, the comparison of data across cases was central to defining these rules (Section 4.1, Tables 4 and 5).

The result of this coding led to the selection of four variables to characterize $\mathrm{BC}$ systems: data veracity control measures, data-entry frequency, smart labels and related customer communication channels and the proportion of accessible data for which hashes are saved on the BC. Two of these variables had already been addressed by previous BCrelated studies (i.e. data veracity control measures, smart labels and communication channels), whereas the other two (i.e. data-entry frequency and the proportion of accessible data for which hashes are saved on the $\mathrm{BC}$ ) had only been briefly mentioned as potential issues in $\mathrm{BC}$ implementations (Table 2). Furthermore, by analysing and comparing cases, we distinguished between different desired levels of upstream and downstream counterfeiting protection (Section 4.2, Tables 4 and 5). This was strictly linked to their exposure to the risk of downstream counterfeiting by potential offenders and the extent to which they intended to insure consumers against upstream counterfeiting, guaranteeing that viticulture and vinification activities followed production and quality standards and were as declared.

As suggested by Miles and Huberman (1994), the data were organized in tabular displays (see Section 4), which was useful for both within-case analyses and cross-case comparisons. They comprised two columns: a description of the case broken down into the relevant variables and the corresponding rating of each variable according to the rules of Table 4.

Cross-case analyses were performed by structuring the data as two-variable matrices (Figure 1) to detect commonalities and differences between cases. The results derived from case analyses were then summarized in the form of propositions.

\section{Case analysis}

\subsection{Blockchain systems}

Case comparison showed the existence of commonalities as well as differences in the implemented BC systems (see Tables 4 and 5).

In all cases, the data were manually uploaded to the $\mathrm{BC}$, but different measures were adopted to control data veracity: from no control measure in cases A and $\mathrm{E}$, to $\mathrm{AI}$ in case D and third-party certification bodies in cases $\mathrm{B}$ and $\mathrm{C}$. While these $\mathrm{BC}$ feeding options are wellknown in the $\mathrm{BC}$ literature (Table 2), an important distinction emerged from the cases concerning data-entry frequency, which had received little attention in previous studies. This varied from some weeks/months (low frequency) in cases $\mathrm{A}$ and $\mathrm{E}$, to some days/one week (medium frequency) in case D and less than $24 \mathrm{~h}$ (high frequency) in cases B and C. This variable appears to be relevant in the characterization of a $\mathrm{BC}$ system because it can influence fraud opportunity. In fact, a higher risk of manipulation occurs when data remain unrecorded and vulnerable to modification for a long period.

As to $\mathrm{BC}$ reading, cases differ in the use of smart labels and customer communication channels, as expected from the $\mathrm{BC}$ literature. In all the examined systems, product-related information displayed to customers through web pages or mobile applications is not taken directly from data saved on the BC. All data are stored on external centralized servers, but customers can verify information provided through the hashes linked to the $\mathrm{BC}$ transactions. This is a common practice, especially when uploading large files. However, a significant difference emerged between cases $\mathrm{A}$ and $\mathrm{E}$ and cases $\mathrm{B}, \mathrm{C}$ and $\mathrm{D}$, regarding the proportion of data for which hashes are saved in the $\mathrm{BC}$. This has important implications for the level of protection assured by $\mathrm{BC}$ technology since a consumer should be able to verify at any time

\section{Designing blockchain systems}


IJOPM

41,13

22

Figure 1.

Cross-case analysis

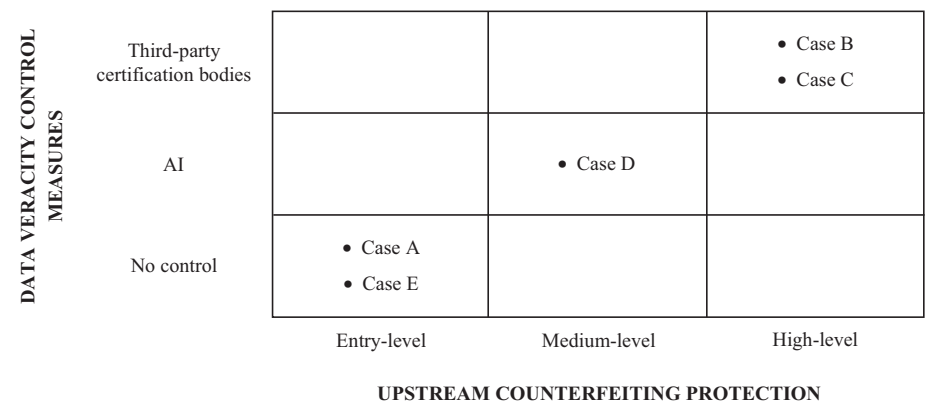

(a)

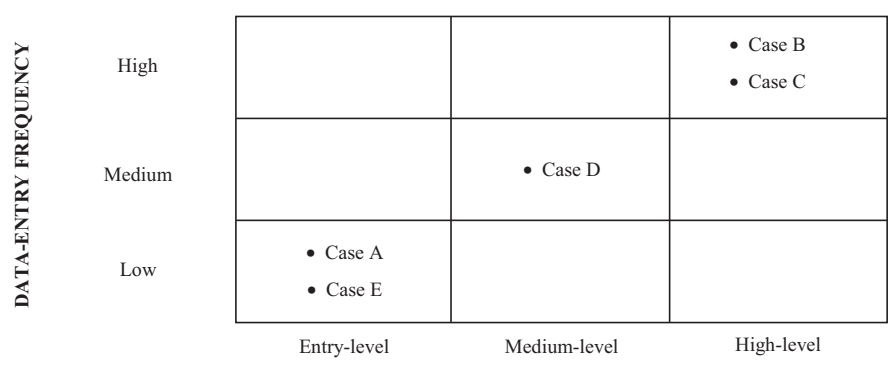

UPSTREAM COUNTERFEITING PROTECTION

(b)

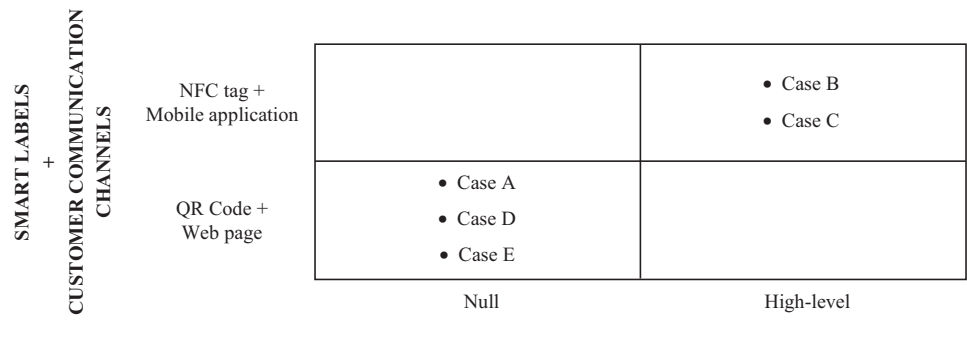

DOWNSTREAM COUNTERFEITING PROTECTION

(c)
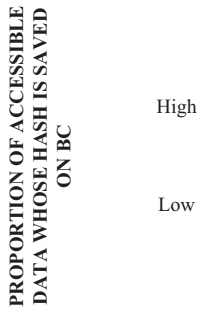

\begin{tabular}{|l|l|l|}
\hline & $\bullet$ Case D & $\bullet$ Case B \\
& & Case C \\
\hline$\cdot$ Case A & & \\
$\bullet$ Case E & & \\
\hline
\end{tabular}

Entry-level Medium-level High-level

UPSTREAM COUNTERFEITING PROTECTION

(d) 
what information is aligned with data originally stored on the $\mathrm{BC}$ to avoid the risk of potential manipulation.

\subsection{Upstream and downstream counterfeiting}

As emerges from Table 5, cases significantly differ in the desired upstream and downstream counterfeiting protection level. This is strictly linked to their business strategy, namely how they intend to create and maintain their competitive advantage through distinctive skills and/ or resources (Mintzberg et al., 1995) and to the risk of downstream counterfeiting depending on their price positioning and target market.

For companies B and C, guaranteeing grape origin, as well as growing and production processes, and product authenticity are of paramount importance. In fact, they own a special know-how related to grape growing and winemaking as well as superior quality assurance skills. They also produce in famous wine areas in compliance with stringent PDO specifications. This results in top-quality wines sold at a very high price, also in international markets. These companies are exposed to the risk of counterfeit copies and thus intend to protect customers against downstream counterfeiting (high level of downstream counterfeiting protection). Moreover, given the special product characteristics, they aim to assure them that wines are produced as declared (high level of upstream counterfeiting protection).

For companies A and E, given the wines they sell (i.e. everyday wines) and consumers they target (i.e. national large supermarkets), there is no real risk of downstream counterfeiting for their products, and thus they are not interested in reducing this risk. They also have no special skills or resources, and thus it is not important for them to provide true empirical evidence of grape origin or adherence to particular production and processing specifications. Instead, they want to reassure customers about the quality and safety of the wine (entry-level of upstream counterfeiting protection).

Finally, for case D, the level of upstream counterfeiting protection the wine producer intends to provide to customers is classified as medium, given its interest in guaranteeing the grape origin on which wine quality differentiation is based. In fact, it produces "niche" everyday wines in a narrow area with morainic hills, and its strategy is based on this distinctive resource, rather than on distinctive production skills. Given the medium-price positioning and target markets (i.e. national large supermarkets), there is no particular risk of downstream counterfeiting (null downstream counterfeiting protection).

\subsection{Formulation of propositions}

In this study, the data were cross-compared to derive propositions for how $\mathrm{BC}$ systems are designed to achieve a desired level of upstream and downstream counterfeiting protection. To this aim, we first investigated the existence of a link between the desired upstream and downstream counterfeiting protection and the $\mathrm{BC}$ system variables (Figure 1). Then, based on the situational crime perspective, which identifies the reduction of the fraud opportunity structure as the key driver in the design of anti-counterfeiting measures, we proposed how the desired degree of counterfeiting protection affects the design of $\mathrm{BC}$ systems given the varying ability of the system variables to reduce fraud opportunity.

The cross-comparison revealed different clusters of cases with similar patterns. Cases A and $\mathrm{E}$, which aimed for an entry-level protection against upstream counterfeiting and no protection against downstream counterfeiting, took similar decisions in terms of BC systems, namely no control measure for data veracity, a low data-entry frequency, the use of QR codes and web pages as customer communication channel, as well as a low proportion of accessible data for which hashes were saved on the BC. Cases B and C exhibit an opposite pattern. They both desired a high level of upstream and downstream counterfeiting protection and adopted

\section{Designing blockchain systems}


IJOPM 41,13

similar BC systems, with third-party certification bodies as data veracity control measure, a high data-entry frequency, NFC tags and mobile applications as customer communication channel, as well as a high proportion of accessible data for which hashes were saved on the $\mathrm{BC}$.

Finally, case D is similar to cases A and E with their use of QR codes and web pages as customer communication channel, while it differs in its use of AI, a medium data-entry frequency and a high proportion of accessible data for which hashes were saved on the BC. Only this latter characteristic is common to cases B and C. Since case D has commonalities with cases $\mathrm{A}$ and $\mathrm{E}$ (no desired downstream counterfeiting protection) and differs from all the cases aiming at a medium level of protection against upstream counterfeiting, it appears evident that the precise relationships between the $\mathrm{BC}$ system options must be considered through a disaggregated analysis to distinguish between upstream and downstream counterfeiting.

The following proposition summarizes the results of the cross-case analysis at an aggregate level:

P1. Different levels of desired upstream and downstream counterfeiting protection determine different $\mathrm{BC}$ systems in terms of (1) data veracity control measures, (2) data-entry frequency, (3) smart label and customer communication channel and (4) proportion of accessible data for which hashes are saved on the $\mathrm{BC}$.

As for data veracity control measures, Figure la suggests that the desired upstream counterfeiting protection level is linked to the use of these measures: from no control when an entry-level of protection is required, to the use of AI when it is at a medium level and the use of third-party certification bodies when it is at a high level.

In cases $\mathrm{B}$ and $\mathrm{C}$, the relevance of making customers aware of the superior quality level of the wine, grape origin and specific production processes led to the use of third-party certification bodies for data collection and data entry. Interviewed managers agreed that this choice entails higher costs, but judged this the best way to dissuade fraudulent behaviour in their context, as it can be employed to control the veracity of a large set of data ranging from process yields to cleaning, cellar temperature and so on. Both cases B and C share a significant amount of information through $\mathrm{BC}$, from cultivation and winemaking processes to information related to the geographical context. The use of third-party certification bodies can insure customers against the risk of deliberate and intentional manipulation of all declared data. In contrast, company $\mathrm{D}$ is mainly interested in providing assurance about the grape origin on which its differentiation is based and thus aims at a medium level of protection to prevent upstream counterfeiting. It adopts AI to detect inconsistencies between some quantitative data (e.g. between hectares cultivated, tonnes of grapes harvested and bottles produced). The existence of inconsistencies means that the wine origin or even quality (e.g. a low percentage of bunch thinning) is not guaranteed; however, their absence offers a certain level of assurance of the use of grapes from declared vineyards. At the same time, AI is ineffective in verifying how some activities have been accomplished (e.g. cleaning of equipment and facilities and proper bottle storage), which is in fact not crucial for a company that aims to differentiate for grape origin rather than production processes. Thus, we can conclude that AI reduces fraud opportunity only to a certain extent and offers a counterfeiting protection level that is lower than the use of third-party certification bodies. Finally, in cases A and E, the desired upstream counterfeiting protection is at an entry-level, as they simply aim to reassure customers that the product quality is acceptable although the price is low, without any interest in providing guarantees for the grape origin or adherence to wine production specifications given that they do not possess distinctive skills and resources. Thus, they do not rely on particular data veracity control measures and can potentially save information on the $\mathrm{BC}$ that does not reflect reality. However, the fact that such information is 
shared through an immutable traceability technology represents an entry-level discouragement of fraudulent conduct.

Based on this evidence, the following proposition is advanced:

P2. The level of desired upstream counterfeiting protection determines the choice of data veracity control measures in the design of $\mathrm{BC}$ systems. To achieve a high level of protection, companies use third-party certification bodies; to achieve a medium level, they use AI; when an entry-level protection is desired no control measures are used.

The pattern in Figure $1 \mathrm{~b}$ is similar. It relates the desired upstream counterfeiting protection to the frequency of entering process-related information on the $\mathrm{BC}$. Cross-case analysis suggests that companies $\mathrm{B}$ and $\mathrm{C}$, aiming for high-level protection, enter data on the $\mathrm{BC}$ with a high frequency. In particular, after a first short period where collected information is stored on the certification body's servers or in the Italian national agricultural information system, data are synchronised with the $\mathrm{BC}$. The whole process takes up to $24 \mathrm{~h}$. This minimizes the time between data collection and data entry during which manipulations may occur, thus reducing the fraud opportunities. For company D, which pursues a medium level of protection, processrelated data are stored and grouped on the technology provider's servers and undergo a further manual correctness check before being immutably saved on the BC. Then, the set of data is registered in a $\mathrm{BC}$ transaction between $24 \mathrm{~h}$ and a week after data collection. When the desired protection is at an entry-level - as in cases A and E - companies first use paper registers or electronic files to group information related to production processes. The timestamps of $\mathrm{BC}$ transactions show significant time lags for events, which range from one week to several months. In general, a higher data-entry frequency implies higher costs, as the number of transactions increases, as in cases B and C. For these latter cases, given the exclusivity of their products, it is important designing a $\mathrm{BC}$ system that offers higher guarantees that no manipulation can occur in the upstream network before on-chain registration, so as to provide customers with strong evidence of their distinctive skills and resources. In summary, we can conclude that the higher the data-entry frequency, the lower the fraud opportunities, as data remain vulnerable to modification for less time. Based on assumptions derived from the situational crime perspective, it follows that a higher dataentry frequency guarantees a higher protection against upstream counterfeiting.

The following proposition encapsulates this evidence:

P3. The level of desired upstream counterfeiting protection determines the choice of dataentry frequency in the design of $\mathrm{BC}$ systems. The higher the level of upstream counterfeiting protection desired, the higher the data-entry frequency.

A further relationship represented in Figure 1c links the desired downstream counterfeiting protection to the different types of smart labels and communication channels. Companies A, $\mathrm{D}$ and $\mathrm{E}$ all use $\mathrm{QR}$ codes printed on the wine label, which redirect to web pages. This solution is cheap, but the interviewed managers are aware that it does not significantly reduce downstream counterfeiting opportunities, as QR codes are also easily cloneable (e.g. by scanning the label, printing it and applying it to a fake bottle). However, in all these cases, downstream counterfeiting is not an issue. The target segment and the price positioning (everyday wines) in cases $\mathrm{A}$ and $\mathrm{E}$ make the risk of products being counterfeited close to zero. Similarly, managers in company $\mathrm{D}$ think that this risk is extremely low given the low brand recognition, the medium-price positioning and the targeted market segment. In contrast, in cases $\mathrm{B}$ and $\mathrm{C}$, the companies decided to use NFC tags with mobile applications. This solution entailed higher costs because it required the redesign of the labelling process. However, the managers agreed that, unlike the QR codes, NFC tags significantly deter downstream counterfeiting, which was a priority for them. In fact, the NFC tags adopted by companies B and $\mathrm{C}$ are difficult to physically remove and provide additional guarantees of non- 
IJOPM

41,13

replicability, such as the encoded unique identifier (UID), as well as data encryption combined with the associated mobile application that decrypts the hidden data stored in the tag. These measures reduce the opportunities for fraudulent conduct, as they discourage counterfeiters from extracting the reference that redirects customers to the web content related to the genuine product from the original NFC tag and then using this link to replicate the tag. Given the exclusivity of their wines due to their distinctive skills and resources, and the consequent need for high-level protection against downstream counterfeiting, companies $\mathrm{B}$ and $\mathrm{C}$ designed a $\mathrm{BC}$ reading solution that is able to provide customers with a stronger guarantee of non-replicability.

The following proposition encapsulates these findings:

P4. The level of desired downstream counterfeiting protection determines the choice of smart label and customer communication channel in the design of $\mathrm{BC}$ systems. To achieve a high level of protection, companies use NFC tags with mobile applications; when no protection is desired, companies use QR codes associated with web pages.

A final important $\mathrm{BC}$ system option regards the proportion of accessible data for which hashes are saved on the BC. For companies B, C and D, which aim for a medium or high level of protection against upstream counterfeiting, most of the data shared with customers is related to the hashes saved on the $\mathrm{BC}$ (Figure 1d). Companies B and C sell high-priced and exclusive wines with a certified grape origin, and their production is complex and meet highquality standards. Company D's differentiation relies on grape cultivation in an exclusive terroir. They are very committed to assuring customers that the shared information is stored on the $\mathrm{BC}$, which offers a higher guarantee that accessible information has not been manipulated. The aim of these companies is to reduce data manipulation and fraud opportunities by giving customers the opportunity to verify the alignment between the information related to their distinctive resources and/or skills and that saved on the BC. Conversely, companies A and $\mathrm{E}$ aim to guarantee an entry-level protection against upstream counterfeiting, as they sell lower priced wines in large-scale supermarket chains and do not rely on distinctive skills and resources. They only share and store information on the $\mathrm{BC}$ that is necessary to guarantee that their wines meet the acceptable quality standards, and then share further information without providing the related hash, assuming that customers are not concerned about adherence to declared production processes or grape origin, which is not distinctive. Thus, compared to the other cases, the restriction of fraud opportunity, and thus the protection level against counterfeiting is lower.

The following proposition summarizes this evidence:

P5. The level of desired upstream counterfeiting protection determines the proportion of accessible data for which hashes are saved on-chain in the design of BC systems. To achieve a high or medium level of protection, the proportion is high; when an entrylevel protection is desired, the proportion is low.

\section{Discussion}

The major theoretical contributions of this study are positioned in the research stream on the design and use of BC technology in supply chains (Treiblmaier, 2018; Queiroz et al., 2019; Wang et al., 2019). This research applies the situational crime perspective to investigate how the degree of protection against counterfeiting a brand owner intends to guarantee to customers affects the design of $\mathrm{BC}$ systems. The use of widely recognized theories to explore the implications of $\mathrm{BC}$ has been advocated by several scholars (Cole et al., 2019; Hald and Kinra, 2019). The interpretation lens of the situational crime perspective, which identifies the restriction of fraud opportunities as the key driver for effective counterfeiting prevention, 
made it possible in the present research to advance knowledge on what is necessary for a more secure $\mathrm{BC}$ system to prevent counterfeiting.

This research contributes to the operationalization of the $\mathrm{BC}$ system concept, including $\mathrm{BC}$ reading and feeding options. The previous literature agrees on the general common key features of a BC technology, such as immutability, traceability, disintermediation and consensus mechanisms (Schmidt and Wagner, 2019; Kumar et al., 2020). Past studies and BC use cases also mention and discuss the possibility that $\mathrm{BC}$ technology could be complemented by different $\mathrm{BC}$ feeding and reading options (Table 2). However, a clear operationalization of the $\mathrm{BC}$ system construct is lacking, and in this regard, the value of the present research is twofold. First, the literature review and comparison with real $\mathrm{BC}$ implementation cases enabled the identification and codification of four relevant variables that can be used to describe a $\mathrm{BC}$ system, namely data veracity control measures, data-entry frequency, smart label and customer communication channel and the proportion of accessible data for which hashes are saved on the BC. Second, the assessment of each BC design option in terms of its protection against upstream and downstream counterfeiting provides researchers and managers with a clearer understanding of the implications of each choice. The situational crime prevention approach (Spink et al., 2013) provided the foundation for the reasoning on the power of each option to prevent counterfeiting by restricting the fraud opportunity structure. Some variables proposed for characterizing a $\mathrm{BC}$ system have already received significant attention in the previous literature; for example, data veracity control measures, which can include the use of AI (Kshetri, 2018; Montecchi et al., 2019; van Hoek, 2019; Bumblauskas et al., 2020; Roeck et al., 2020), or neutral third-party bodies for the verification of data quality (Creydt and Fischer, 2019) or smart labels and customer communication channels differentiated by the use of common tags like QR codes associated with a web page (Azzi et al., 2019; Lo et al., 2019) and more sophisticated methods like NFC tags associated with a mobile application (Alzahrani and Bulusu, 2018, 2019). As expected, the present research confirms that decisions on smart labels and customer communication channels are determined by the desired downstream counterfeiting protection level, as they use different security measures (e.g. UID and data encryption) characterized by varying dissuading power against fraudulent actions such as cloning or re-labelling (Alzahrani and Bulusu, 2018). As to data veracity control measures, instead, based on case studies, this research elaborates interesting arguments and novel evidence on the level of protection against upstream counterfeiting that AI can guarantee compared to neutral third-party bodies, which were considered more effective as they allow verifying more information beyond quantitative data. Although this evidence requires further research (see Section 6.1), it may contribute to stimulating a more in-depth investigation of the use of AI to understand under what conditions their potential is fully exploited. This research also identifies two variables characterizing a $\mathrm{BC}$ system that received less attention in previous studies: data-entry frequency and the proportion of accessible data for which hashes are saved on the BC. Although the issues of data-entry frequency (Zhang et al., 2020) and the common practice of not making information visible to final customers directly from the $\mathrm{BC}$ are mentioned in the $\mathrm{BC}$ literature (Singhal et al., 2018), this research is more precise in identifying the differences that can exist between cases, which is important as the ability to reduce fraud opportunities (and thus the upstream counterfeiting protection level) can be significantly affected. Dataentry frequency can vary from data written on the BC from less than $24 \mathrm{~h}$ to months, greatly changing the risk of data manipulation. Similarly, when most of the information provided to consumers is linked to the respective transactions saved permanently on the $\mathrm{BC}$, companies are providing customers the opportunity to verify the data, significantly reducing fraud opportunities and providing greater protection against upstream counterfeiting.

Overall, these results further contribute to the research on the design and use of $\mathrm{BC}$ technology by emphasizing the importance of the organizational dimension in designing $\mathrm{BC}$

\section{Designing blockchain systems}


IJOPM 41,13

systems. Some authors (Risius and Spohrer, 2017; Constantinides et al., 2018; Pereira et al., 2019) warn about the prevalent focus on the technical aspects of $\mathrm{BC}$ (e.g. type of permission model, consensus mechanisms, block structure, etc.) and their interaction with technological tools (e.g. RFID, NFC tags, IoT, etc.), while an investigation from an organizational and managerial perspective is less explored. The present research indicates that some decisions on the processes related to $\mathrm{BC}$ feeding and reading, such as the choice of involving third-party certification bodies, the proportion of accessible data for which hashes are saved on the $\mathrm{BC}$ and data-entry frequency, are crucial in determining the power of a $\mathrm{BC}$ system for preventing counterfeiting.

This study contributes to the debate on the potential of $\mathrm{BC}$ technology to guarantee product authenticity in comparison to traditional approaches, both in general and in particular in the wine industry (see, for example, Apte and Petrovsky, 2016; Biswas et al., 2017; Alzahrani and Bulusu, 2018). Previous scholars (e.g. Galvez et al., 2018; Lu et al., 2019) agree that $\mathrm{BC}$ might be considered a promising solution for effectively preventing product counterfeiting because of its characteristics, but did not investigate the validity of such an assumption in real cases. The conclusion that different $\mathrm{BC}$ system configurations are related to different protection levels against upstream and downstream counterfeiting, the classification of the different $\mathrm{BC}$ system options accounting for $\mathrm{BC}$ feeding and reading processes and the detailed explanation of how the desired degree of counterfeiting protection affects the design of $\mathrm{BC}$ systems pave the way for a clearer and more objective comparison with existing measures for counterfeiting. In particular, our work suggests that BC systems have a wider scope than other measures (except for track-and-trace systems) since each of them is focused on preventing a single type of counterfeiting, whereas BC systems can be used to prevent both upstream and downstream counterfeiting (see Tables 1 and 4). Moreover, this study claims that any comparison between the characteristics of $\mathrm{BC}$ systems and other counterfeiting measures should be made at a micro rather than macro level, namely considering the characteristics of each specific BC system. In theory, a measure that only focuses on upstream counterfeiting (e.g. a measure aimed at authenticating product consistency with production regulations using external auditors that collect on-field evidence) could provide higher guarantees than a $\mathrm{BC}$ characterized by an entry-level protection against upstream counterfeiting, while only a $\mathrm{BC}$ system designed to fully exploit its potential in reducing fraud opportunities both upstream and downstream actually offers a high degree of confidence in the prevention of both types of counterfeiting. Previous studies (Apte and Petrovsky, 2016; Biswas et al., 2017; Alzahrani and Bulusu, 2018) discuss how BC implementation is expected to contrast counterfeiting and overcome some weaknesses of traditional counterfeiting measures (Table 1), by reducing the risk of post-data-entry manipulations and allowing an "average" user to autonomously verify the authenticity of each product without the need to involve specific equipment/competences or perform destructive chemical/physical/organoleptic analyses. This research instead suggests that assessing $\mathrm{BC}$ protection levels against upstream and downstream counterfeiting requires careful consideration of $\mathrm{BC}$ feeding and reading options since they can influence $\mathrm{BC}$ performance in terms of fraud opportunity restriction.

\subsection{Managerial implications}

This study provides some practical suggestions to properly address the design and use of BC systems.

First, it warns managers against using the $\mathrm{BC}$ as a stand-alone technology and instead suggests viewing it as a part of a wider $\mathrm{BC}$ system where decisions on technical aspects (e.g. type of smart labels and communication channels, use of AI, etc.) must be complemented with organisational choices regarding $\mathrm{BC}$ feeding and reading processes (e.g. third-party 
involvement and data-entry frequency). A coherent set of these decisions is crucial to ensuring that data written in and read from the $\mathrm{BC}$ accurately reflects reality.

Second, the study suggests that $\mathrm{BC}$ systems are not one-size-fits-all solutions and therefore should be adapted to the required upstream and/or downstream counterfeiting protection level. Specifically, the study presents a set of variables that managers should consider when designing $\mathrm{BC}$ systems and shows how they can be modulated in accordance with the desired guarantees of product authenticity. Several key learnings for managers can

be derived. This research indicates how to design $\mathrm{BC}$ systems characterized by a high level of protection against upstream and/or downstream counterfeiting. It also argues that in certain contexts (as in cases $\mathrm{A}$ and $\mathrm{E}$ ), the use of $\mathrm{BC}$ systems without particular attention to strict data input and reading measures is a good solution, as they offer some deterrent power against intentional infringements and also operate as traceability and communication tools that allow customers to autonomously verify the safety of products they buy. For instance, in the case of upstream counterfeiting, an entry-level protection is better than no protection since the core technical features of BC technology (e.g. transparency, immutability and traceability) allow the discovery of post-entry manipulation and the detection of the counterfeiter, thus making offending less feasible or attractive. In the case of downstream counterfeiting, no protection can be a viable choice where there is no real risk of downstream counterfeiting (e.g. low price and no brand products).

The third practical contribution is directed towards policymakers who are evaluating the potential of $\mathrm{BC}$ vs existing measures to protect consumers, honest producers and national brands from risks due to fraudulent copies or imitations. This study corroborates the advisability of the prudential decisions of many governments to fund BC-based pilot projects to explore the real potential of this technology to defend product authenticity before extending its use on a large scale. However, it also suggests caution in considering $\mathrm{BC}$ a panacea that overcomes all the weaknesses of existing measures. This study demonstrates that $\mathrm{BC}$ systems are versatile, their real potential in reducing fraud opportunities (and defending product authenticity) in comparison with existing measures can vary and that choices on $\mathrm{BC}$ feeding and reading determine this variation. Thus, the configuration and management of such processes is crucial to ensure that BC technology is better able to guarantee product authenticity than existing measures.

\section{Conclusions}

This research represents a first attempt to apply the situational crime perspective to the study of $\mathrm{BC}$ systems, with a focus on the different options of $\mathrm{BC}$ feeding and reading that complement the use of $\mathrm{BC}$ technology. Based on the analysis of five case studies in the wine sector, this paper concludes that the desired upstream and downstream counterfeiting protection level guides decisions on $\mathrm{BC}$ system configurations, as their ability to reduce fraud opportunities is variable. In particular, this research advances that the desired upstream counterfeiting protection level determines choices of data veracity control measures, data-entry frequency and the proportion of accessible data for which hashes are saved on the $\mathrm{BC}$; whereas the desired downstream counterfeiting protection level determines decisions on smart labels and customer communication channels. The higher the desired protection level, the more the companies choose those $\mathrm{BC}$ options that can reduce fraud opportunities to a greater extent.

\subsection{Limitations and future research directions}

A first limitation of this study is linked to the characteristics of the cases investigated (Section 3.1.1), which restricts the generalizability of this research and sets precise research boundaries. First, as in most existing $\mathrm{BC}$ initiatives, all the examined $\mathrm{BC}$ projects are in the

\section{Designing blockchain systems}


IJOPM 41,13

pilot phase and thus involve a limited number of companies and products. Further studies may consider $\mathrm{BC}$ system implementations in more complex supply networks. For instance, a possible issue may concern the scalability of the use of third-party physical inspections, as in cases B and C. Whereas cost is not significant in simple supply chains such as those analysed in this study, third-party inspections could become economically unsustainable in more complex supply networks. Second, all the analysed cases apply public BC technologies that differ significantly from private BCs in terms of data immutability and decentralized governance, and thus conceptually in their counterfeiting protection. Further studies of private $\mathrm{BCs}$ are needed to understand the effectiveness of their adoption for anticounterfeiting and whether and how $\mathrm{BC}$ systems may differ in terms of $\mathrm{BC}$ feeding and reading. Third, as argued in Section 3.1.1, the implementation of the IoT in the wine sector is still a challenge. Future research could investigate the issue of $\mathrm{BC}$ feeding in more automated sectors, such as the pharmaceutical sector, to understand the implications of IoT use and its efficacy in solving BC feeding issues. Although several scholars (e.g. Kamble et al., 2019; Bumblauskas, 2020) consider the IoT an effective means of guaranteeing objective data collection and recording, which reduces the risk of human manipulation; others argue that IoT sensors can also be subject to intentional manipulation (Rejeb et al., 2019; Schmidt and Wagner, 2019). In the future, more specific IoT sensors could be developed and spread to appropriately detect important production data, e.g. in the wine cellars. Further research, in the wine or luxury clothing contexts, could investigate the use of the IoT in combination with $\mathrm{AI}$ algorithms to support BC feeding. These solutions could eliminate the need for third-party certification bodies for data that are currently not measurable by sensors.

A further limitation of this study is that it assumes the perspective of a brand owner who configures the $\mathrm{BC}$ system in line with a desired counterfeiting protection level. Once $\mathrm{BC}$ systems become more mature and widely used, it will become essential to consider the buyers' perspective, in particular the final customers' perspective, to understand to what extent and for which clusters of customers the perceived risk of counterfeiting results is reduced through $\mathrm{BC}$ technology.

\section{References}

Ali, M.H., Zhan, Y., Alam, S.S., Tse, Y.K. and Tan, K.H. (2017), "Food supply chain integrity: the need to go beyond certification”, Industrial Management and Data Systems, Vol. 117 No. 8, pp. 1589-1611.

Alzahrani, N. and Bulusu, N. (2018), "Block-supply chain: a new anti-counterfeiting supply chain using NFC and blockchain”, 1st Workshop on Cryptocurrencies and Blockchains for Distributed Systems, pp. 30-35.

Alzahrani, N. and Bulusu, N. (2019), "A new product anti-counterfeiting blockchain using a truly decentralized dynamic consensus protocol", Concurrency and Computation: Practice and Experience, Vol. 32 No. 12, pp. 1-27.

Apte, S. and Petrovsky, N. (2016), "Will blockchain technology revolutionize excipient supply chain management?", The Journal of Excipients and Food Chemicals, Vol. 7 No. 3, pp. 76-78.

Azzi, R., Chamoun, R.K. and Sokhn, M. (2019), "The power of a blockchain-based supply chain", Computers and Industrial Engineering, Vol. 135, pp. 582-592.

Babich, V. and Hilary, G. (2019), "Distributed ledgers and operations: what operations management researchers should know about blockchain technology", Manufacturing and Service Operations Management, Vol. 22 No. 2, pp. 223-240.

Berman, B. (2008), "Strategies to detect and reduce counterfeiting activity", Business Horizons, Vol. 51 No. 3, pp. 191-199. 
Biswas, K., Muthukkumarasamy, V. and Tan, W.L. (2017), "Blockchain based wine supply chain traceability system", Paper Presented at Future Technologies Conference (FTC) 2017, 29-30 Nov, Vancouver, Canada, available at: https://saiconference.com/Conferences/FTC2017 Proceedings (accessed 10 February 2021).

Designing blockchain systems

Bowen, G.A. (2008), "Naturalistic inquiry and the saturation concept: a research note", Qualitative Research, Vol. 8 No. 1, pp. 137-152.

Bumblauskas, D., Mann, A., Dugan, B. and Rittmer, J. (2020), "A blockchain use case in food distribution: do you know where your food has been?", International Journal of Information Management, Vol. 52, 102008.

Cole, R., Stevenson, M. and Aitken, J. (2019), "Blockchain technology: implications for operations and supply chain management”, Supply Chain Management: An International Journal, Vol. 24 No. 4, pp. 469-483.

Constantinides, P., Henfridsson, O. and Parker, G.G. (2018), "Introduction - platforms and infrastructures in the digital age", Information Systems Research, Vol. 29 No. 2, pp. 381-400.

Creswell, J.W. (2013), Qualitative Inquiry and Research Design: Choosing Among Five Approaches, Sage Publications, Thousand Oaks, California.

Creydt, M. and Fischer, M. (2019), "Blockchain and more - algorithm driven food traceability", Food Control, Vol. 105, pp. 45-51.

Eisenhardt, K.M. and Graebner, M.E. (2007), “Theory building from cases: opportunities and challenges", Academy of Management Journal, Vol. 50 No. 1, pp. 25-32.

EUIPO (2019), “Anti-counterfeiting blockathon forum”, available at: https:/euipo.europa.eu/ ohimportal/en/web/observatory/blockathon (accessed June 2020).

Galvez, J.F., Mejuto, J.C. and Simal-Gandara, J. (2018), "Future challenges on the use of blockchain for food traceability analysis", Trends in Analytical Chemistry, Vol. 107, pp. 222-232.

Hald, K.S. and Kinra, A. (2019), "How the blockchain enables and constrains supply chain performance", International Journal of Physical Distribution and Logistics Management, Vol. 49 No. 4, pp. 376-397.

Hopkins, D.M., Kontnik, L.T. and Turnage, M.T. (2003), Counterfeiting Exposed: How to Protect Your Brand and Market Share Hoboken, John Wiley and Sons, New Jersey.

Kamble, S., Gunasekaran, A. and Arha, H. (2019), "Understanding the blockchain technology adoption in supply chains-Indian context", International Journal of Production Research, Vol. 57 No. 7 , pp. 2009-2033.

Kamilaris, A., Fonts, A. and Prenafeta-Boldú,F.X.(2019), “Therise of blockchain technology in agriculture and food supply chains", Trends in Food Science and Technology, Vol. 91, pp. 640-652.

Kshetri, N. (2018), "Blockchain's roles in meeting key supply chain management objectives", International Journal of Information Management, Vol. 39, pp. 80-89.

Kumar, A., Liu, R. and Shan, Z. (2020), "Is blockchain a silver bullet for supply chain management? Technical challenges and research opportunities”, Decision Sciences, Vol. 51 No. 1, pp. 8-37.

Li, L. (2013), "Technology designed to combat fakes in the global supply chain”, Business Horizons, Vol. 56 No. 2, pp. 167-177.

Lo, S.K., Xu, X., Wang, C., Weber, I., Rimba, P., Lu, Q. and Staples, M. (2019), "Digital-physical parity for food fraud detection”, in Joshi, J., Nepal, S., Zhang, Q. and Zhang, L.J. (Eds), Blockchain ICBC 2019, Lecture Notes in Computer Science, Vol. 11521, Springer, Cham, pp. 65-79.

Lu, D., Moreno-Sanchez, P., Zeryihun, A., Bajpayi, S., Yin, S., Feldman, K., Kosofsky, J., Mitra, P. and Kate, A. (2019), "Reducing automotive counterfeiting using blockchain: benefits and challenges", IEEE International Conference on Decentralized Applications and Infrastructures, pp. 39-48.

Lybecker, K.M. (2007), "Rx roulette: combatting counterfeit pharmaceuticals in developing nations", Managerial and Decision Economics, Vol. 28 Nos 4/5, pp. 509-520. 
IJOPM 41,13

Martinez, V., Zhao, M., Blujdea, C., Han, X., Neely, A. and Albores, P. (2019), "Blockchain-driven customer order management", International Journal of Operations and Production Management, Vol. 39 Nos 6/7/8, pp. 993-1022.

Miles, M.B. and Huberman, A.M. (1994), Qualitative Data Analysis: An Expanded Sourcebook, Sage Publications, Thousand Oaks, California.

Mintzberg, H., Quinn, J.B. and Ghoshal, S. (1995), The Strategy Process, Prentice Hall International, Hemel Hampstead.

Montecchi, M., Plangger, K. and Etter, M. (2019), "It's real, trust me! Establishing supply chain provenance using blockchain”, Business Horizons, Vol. 62 No. 3, pp. 283-293.

Pandey, S.C. and Patnaik, S. (2014), "Establishing reliability and validity in qualitative inquiry: a critical examination", Journal of Development and Management Studies, Vol. 12 No. 1, pp. 5743-5753.

Pereira, J., Tavalaei, M.M. and Ozalp, H. (2019), "Blockchain-based platforms: decentralized infrastructures and its boundary conditions", Technological Forecasting and Social Change, Vol. 146, pp. 94-102.

Pustjens, A.M., Weesepoel, Y. and van Ruth, S.M. (2016), "Food fraud and authenticity: emerging issues and future trends", in Leadley, C. (Ed.), Innovation and Future Trends in Food Manufacturing and Supply Chain Technologies, Woodhead Publishing, Cambridge, pp. 3-20.

Queiroz, M.M., Telles, R. and Bonilla, S.H. (2019), "Blockchain and supply chain management integration: a systematic review of the literature", Supply Chain Management: An International Journal, Vol. 25 No. 5, pp. 241-254.

Rejeb, A., Keogh, J.G. and Treiblmaier, H. (2019), "Leveraging the internet of things and blockchain technology in supply chain management”, Future Internet, Vol. 11 No. 7, pp. 1-22.

Risius, M. and Spohrer, K. (2017), "A blockchain research framework", Business and Information Systems Engineering, Vol. 59 No. 6, pp. 385-409.

Roeck, D., Sternberg, H. and Hofmann, E. (2020), "Distributed ledger technology in supply chains: a transaction cost perspective", International Journal of Production Research, Vol. 58 No. 7, pp. 2124-2141.

Saberi, S., Kouhizadeh, M., Sarkis, J. and Shen, L. (2019), "Blockchain technology and its relationships to sustainable supply chain management", International Journal of Production Research, Vol. 57 No. 7, pp. 2117-2135.

Schmidt, C.G. and Wagner, S.M. (2019), "Blockchain and supply chain relations: a transaction cost theory perspective", Journal of Purchasing and Supply Management, Vol. 25 No. 4, 100552.

Singhal, B., Dhameja, G. and Panda, P.S. (2018), Beginning Blockchain: A Beginner's Guide to Building Blockchain Solutions, Apress, New York, New York.

Speier, C., Whipple, J.M., Closs, D.J. and Voss, M.D. (2011), "Global supply chain design considerations: mitigating product safety and security risks", Journal of Operations Management, Vol. 29 Nos 7-8, pp. 721-736.

Spink, J., Moyer, D.C., Park, H. and Heinonen, J.A. (2013), "Defining the types of counterfeiters, counterfeiting and offender organizations", Crime Science, Vol. 2 No. 8, pp. 1-10.

Stevenson, M. and Busby, J. (2015), "An exploratory analysis of counterfeiting strategies", International Journal of Operations and Production Management, Vol. 35 No. 1, pp. 110-144.

Treiblmaier, H. (2018), "The impact of the blockchain on the supply chain: a theory-based research framework and a call for action”, Supply Chain Management: An International Journal, Vol. 23 No. 6, pp. 545-559.

van Hoek, R. (2019), "Exploring blockchain implementation in the supply chain”, International Journal of Operations and Production Management, Vol. 39 Nos 6/7/8, pp. 829-859.

Villano, C., Lisanti, M.T., Gambuti, A., Vecchio, R., Moio, L., Frusciante, L., Aversano, R. and Carputo, D. (2017), "Wine varietal authentication based on phenolics, volatiles and DNA markers: state of the art, perspectives and drawbacks", Food Control, Vol. 80, pp. 1-10. 
Vinex (2020), "Massive wine fraud uncovered by French trading standards", available at: https:/en. vinex.market/articles/2018/03/19/massive_wine_fraud_uncovered_by_french_trading_ standard (accessed September 2020).

Viriyasitavat, W. and Hoonsopon, D. (2019), "Blockchain characteristics and consensus in modern business processes", Journal of Industrial Information Integration, Vol. 13, pp. 32-39.

Voss, C., Johnson, M. and Godsell, J. (2016), “Case research”, in Karlsson, C. (Ed.), Research Methods for Operations Management, Routledge, London, pp. 165-197.

Wamba, S.F. and Queiroz, M.M. (2020), "Blockchain in the operations and supply chain management:

Designing blockchain systems benefits, challenges and future research opportunities", International Journal of Information Management, Vol. 52, 102064.

Wang, Y., Han, J.H. and Beynon-Davies, P. (2019), "Understanding blockchain technology for future supply chains: a systematic literature review and research agenda", Supply Chain Management: An International Journal, Vol. 24 No. 1, pp. 62-84.

Wine Folly (2016), "Reality of wine prices (what you get for what you spend)", available at: https:// winefolly.com/update/reality-of-wine-prices-what-you-get-for-what-you-spend/ (accessed June 2020).

World Trade Organization (2020), “Glossary: counterfeit definition”, available at: https://www.wto.org/ english/thewto_e/glossary_e/glossary_e.htm (accessed September 2020).

Xu, X., Lu, Q., Liu, Y., Zhu, L., Yao, H. and Vasilakos, A.V. (2019), "Designing blockchain-based applications a case study for imported product traceability", Future Generation Computer Systems, Vol. 92, pp. 399-406.

Yin, R.K. (2017), Case Study Research and Applications: Design and Methods, Sage Publications, Thousand Oaks, California.

Zelbst, P.J., Green, K.W., Sower, V.E. and Bond, P.L. (2019), "The impact of RFID, IIoT, and blockchain technologies on supply chain transparency", Journal of Manufacturing Technology Management, Vol. 31 No. 3, pp. 441-457.

Zhang, A., Zhong, R.Y., Farooque, M., Kang, K. and Venkatesh, V.G. (2020), "Blockchain-based life cycle assessment: an implementation framework and system architecture", Resources, Conservation and Recycling, Vol. 152, 104512.

\section{Appendix}

Supplementary file 1 on available online

\section{Corresponding author}

Pamela Danese can be contacted at: pamela.danese@unipd.it

For instructions on how to order reprints of this article, please visit our website:

www.emeraldgrouppublishing.com/licensing/reprints.htm

Or contact us for further details: permissions@emeraldinsight.com 